Check for updates

Cite this: RSC Adv., 2018, 8, 27448

\section{Combined systems pharmacology and fecal metabonomics to study the biomarkers and therapeutic mechanism of type 2 diabetic nephropathy treated with Astragalus and Leech $\dagger$}

\author{
Ruiqun Chen,,$_{+}^{\mathrm{a}}$ Chengbin Liao, $\stackrel{+}{\mathrm{a}}^{\mathrm{a}}$ Qian Guo, ${ }^{a}$ Lirong $\mathrm{Wu},{ }^{\mathrm{b}}$ Lei Zhang ${ }^{\mathrm{a}}$ \\ and Xiufeng Wang (D)*a
}

In our study, systems pharmacology was used to predict the molecular targets of Astragalus and Leech, and explore the therapeutic mechanism of type 2 diabetic nephropathy (T2DN) treated with Astragalus and Leech. Simultaneously, to reveal the systemic metabolic changes and biomarkers associated with T2DN, we performed ${ }^{1} \mathrm{H}$ NMR-based metabonomics and multivariate analysis to analyze fecal samples obtained from model T2DN rats. In addition, ELISA kits and histopathological studies were used to examine biochemical parameters and kidney tissue, respectively. Striking differences in the Pearson's correlation of 22 biomarkers and 9 biochemical parameters were also observed among control, T2DN and treated rats. Results of systems pharmacology analysis revealed that 9 active compounds (3,9-di-Omethylnissolin; $\quad(6 a R, 11 a R)-9,10-$ dimethoxy-6a,11a-dihydro-6H-benzofurano[3,2-c]chromen-3-ol; hirudin; L-isoleucine; phenylalanine; valine; hirudinoidine $\mathrm{A}-\mathrm{C}$ ) and 9 target proteins ( $\mathrm{L}$-serine dehydratase; 3-hydroxyacyl-CoA dehydrogenase; tyrosyl-tRNA synthetase; tryptophanyl-tRNA synthetase; branched-chain amino acid aminotransferase; acetyl-COA C-acetyltransferase; isovalerylCoA dehydrogenase; pyruvate dehydrogenase E1 component alpha subunit; hydroxyacylglutathione hydrolase) of Astragalus and Leech were closely associated with the treatment of T2DN. Using fecal metabonomics analysis, 22 biomarkers were eventually found to be closely associated with the occurrence of T2DN. Combined with systems pharmacology and fecal metabonomics, these biomarkers were found to be mainly associated with 6 pathways, involving amino acid metabolism (leucine, valine, isoleucine, alanine, lysine, glutamate, taurine, phenylalanine, tryptophan); energy metabolism (lactate, succinate, creatinine, $\alpha$-glucose, glycerol); ketone body and fatty acid metabolism (3-hydroxybutyrate, acetate, $n$-butyrate, propionate); methylamine metabolism (dimethylamine, trimethylamine); and secondary bile acid metabolism and urea cycle (deoxycholate, citrulline). The underlying mechanisms of action included protection of the liver and kidney, enhancement of insulin sensitivity and antioxidant activity, and improvement of mitochondrial function. To the best of our knowledge, this is the first time that systems pharmacology combined with fecal metabonomics has been used to study T2DN. 6 metabolites ( $n$-butyrate, deoxycholate, propionate, tryptophan, taurine and glycerol) associated with T2DN were newly discovered in fecal samples. These 6 metabolites were mainly derived from the intestinal flora, and related to amino acid metabolism, fatty acid metabolism, and secondary bile acid metabolism. We hope the results of this study could be inspirational and helpful for further exploration of T2DN treatment. Meanwhile, our results highlighted that exploring the biomarkers of T2DN and therapeutic mechanisms of Traditional Chinese Medicine (TCM) formulas on T2DN by combining systems pharmacology and fecal metabonomics methods was a promising strategy.
Received 22nd May 2018 Accepted 19th July 2018

DOI: $10.1039 / \mathrm{c} 8 \mathrm{ra0} 4358 \mathrm{~b}$

rsc.li/rsc-advances

\section{Introduction}

${ }^{a}$ School of Basic Courses, Guangdong Pharmaceutical University, Guangzhou 510006, P. R. China. E-mail: wxfsnow8012@126.com; Fax: +86-20-39352186; Tel: +86-2039352195

${ }^{b}$ Guangdong Provincial Key Laboratory of Pharmaceutical Bioactive Substances, Guangdong Pharmaceutical University, Guangzhou 510006, P. R. China

$\dagger$ Electronic supplementary information (ESI) available. See DOI: 10.1039/c8ra04358b

\$ Co-first authors.
Diabetic nephropathy (DN), a major microvascular complication of diabetes mellitus (DM), is characterized by the progressive impairment of glomerular filtration and high blood glucose levels leading to end-stage renal failure (ESRD). ${ }^{1}$ It occurs in approximately $20-40 \%$ of patients with type 2 diabetes 
mellitus (T2DM) and has been the most common cause of ESRD and a major health problem worldwide. ${ }^{2}$ A growing body of evidence has demonstrated that the pathogenesis of $\mathrm{DN}$ is complex. It is the result of multi-factor and multi-pathway synergy, including renal hemodynamic changes, the accumulation of advanced glycation end products (AGEs) and reactive oxygen species (ROS), activation of transforming growth factor (TGF- $\beta_{1}$ ), mitogen activated protein kinase (MAPK) signaling pathway and renin-angiotensin system (RAS), etc. ${ }^{3-6}$ Thus, there is an urgent need for a novel approach to comprehensively investigate the underlying mechanism and systemic changes related to T2DN. At present, due to the modeling stability, high animal survival rate and low tissue toxicity, streptozotocin (STZ)-induced DN rats' pancreatic injury are extensively used for creating the rodent model which develops renal injury with similarities to human DN. ${ }^{7}$

Metabonomics analysis is a systems biology approach that provides a wealth of metabolic information in biological samples rapidly, and has been widely used in the diagnosis and evaluation of diabetes and diabetic nephropathy. ${ }^{8-10}$ Much of the original study related with DN metabonomics are performed using nuclear magnetic resonance (NMR) spectroscopy, gas chromatography (GC) or liquid chromatography (LC) coupled with mass spectrometry (MS), but most of them are concentrated in several biological samples, such as serum/ plasma, urine and renal extracts. ${ }^{11-13}$ Feces are also suitable for high-throughput analytical methods and this approach has not yet, to our knowledge, been applied to the metabonomic analysis of T2DN. ${ }^{14}$ Studies have shown that ${ }^{1} \mathrm{H}$ NMR spectral profiles of fecal extracts carry diagnostic information for diseases, and it is potential to use fecal metabolites in the study of renal damage. ${ }^{15,16}$

Traditional Chinese medicine (TCM) has been practiced for thousands of years in China with increasing awareness of its application in the prevention and treatment of complex multifactor diseases (e.g. DN) through multi-components, multipathways and multi-targets. ${ }^{17,18}$ Astragalus (Huangqi), a kind of TCM firstly recorded in "Shen Nong's Herbal Classic", is a common herb for the treatment of diabetes in traditional Chinese medicine and has the highest frequency of use in the TCM formulas of diabetes. ${ }^{19,20}$ Modern pharmacological studies have shown that Astragalus contains a variety of active ingredients, including Astragalus polysaccharides, amino acids, saponins, $\gamma$-aminobutyric acid, and flavonoids, such as calycosin and formononetin. ${ }^{19,21} \mathrm{~A}$ wealth of literatures have shown that Astragalus can protect DN by various mechanisms such as antiperoxidation, regulating the balance of endothelin (ET) and nitric oxide (NO), and improving the glomerular filtration barrier, etc. ${ }^{19,22-26}$ Leech (Shuizhi), also a kind of TCM firstly recorded in "Shen Nong's Herbal Classic", is used commonly for activating blood circulation to dissipate blood stasis. ${ }^{27,28}$ It mainly contains protein, hirudin and various trace elements and amino acids, ${ }^{29,30}$ and has been extensively studied for their anticoagulant compounds which maintain the blood in a fluid state during the uptake and in their guts. Reports have shown that Leech can change the renal hemodynamics, increase renal blood flow and glomerular filtration rate, so as to achieve the purpose of recovery of renal function, and the treatment of DN. ${ }^{31}$ What's more, studies have also shown that Leech capsule has a significant effect on the hemorheology and urinary protein excretion in patients with diabetic nephropathy in different stages. ${ }^{32}$ On one hand, it has been reported that the combination of Astragalus and Leech can effectively treat nephropathy, and the effect is better than the administration of Astragalus or Leech alone. ${ }^{33,34}$ On the other hand, according to the theory of TCM, Qi deficiency and blood stasis throughout the whole process of DN's occurrence and development, is the main cause of DN incidence. ${ }^{35}$ Astragalus and Leech, representatives of benefiting Qi, and promoting blood circulation and removing blood stasis respectively in TCM, are widely used to treat DN. ${ }^{19}$ Simultaneously, clinical studies have shown that Huangqi Shuizhi mixture have significant effects on early diabetic nephropathy. ${ }^{36}$ Some studies also have shown that the Huangqi Shuizhi preparation may improve the renal function by inhibiting the inflammation of DN rats and reducing the occurrence of proteinuria. ${ }^{37,38}$ Hence, Astragalus and Leech are combined to treat T2DN rats and its efficacy is further evaluated in this study. Finally, in order to gain a deeper understanding of the active compounds and targets of Astragalus and Leech to improve diabetic nephropathy, we perform a rapid screening with systems pharmacology. Systems pharmacology is an emerging field that integrates systems biology and pharmacology to advance the process of drug discovery, development and the understanding of therapeutic mechanisms. ${ }^{39}$ What's more, metabolomic approach together with the systems pharmacology approach has been widely used to study a system (e.g. TCM formula). ${ }^{40-42}$

Taken together, in the study, systems pharmacology combined with ${ }^{1} \mathrm{H}$ NMR-based fecal metabonomics is firstly used to analyze the metabolic changes in feces from type 2 diabetic nephropathy rats induced by streptozotocin and highfat diet.

\section{Materials and methods}

\section{Chemicals and reagents}

Streptozocins (STZ) were purchased from Sigma-Aldrich (MO, USA). The urinary creatinine and $24 \mathrm{~h}$ urinary protein excretion quantitative assay kits were purchased from Nanjing Jiancheng Bioengineering Institute (Nanjing, China). Wenhao blood glucose meter and glucose test strips (glucose oxidase method) were provided by Johnson \& Johnson (One Touch Ultra, Lifescan). D12451 high-fat diet (Research Diets, USA) was bought by entrusting Guangdong Medical Experimental Animal Center. $5 \mathrm{~mm}$ nuclear magnetic resonance (NMR) tube and deuterium oxide $\left(\mathrm{D}_{2} \mathrm{O}\right)$ was purchased from Qingdao Teng Long Technology Co., LTD (Qingdao, China). BS180 automatic biochemistry analyzer and other biochemical assay kits were provided by Mindray Bio-medical electronics Co., LTD (Shenzhen, China). $500 \mathrm{MHz}$ Bruker AVANCE III NMR instrument (Bruker, Swiss) was provided by Center Laboratory of Guangdong Pharmaceutical University. Visible spectrophotometer was got from Shanghai Lengguang Technology Co., LTD (Shanghai, China). Distilled water (18.2 M $\Omega$ ) was obtained from a Milli-Q water 
purification system (Millipore, MA, USA). Astragalus (Huangqi, batch number: YPA7C0001) and Leech (Shuizhi, batch number: YPA7B0001) in this study were purchased from Chinese Medicine Yinpian Factory of Guangzhou Medicine Company (Guangzhou, China), and authenticated by associate professor Hongyan Ma (College of Traditional Chinese Medicine of Guangdong Pharmaceutical University). The identification work were mainly based on the experience of associate professor Hongyan Ma for many years in the identification of medicinal materials, and the shape, size, color, surface, section and smell characteristics of Astragalus and Leech.

\section{Preparation of Astragalus and Leech}

$180 \mathrm{~g}$ Astragalus were soaked in $500 \mathrm{~mL}$ distilled water for $0.5 \mathrm{~h}$ in $1000 \mathrm{~mL}$ round bottom flask and heated to reflux for $0.5 \mathrm{~h}$, and then filtration with five layers of gauze. The filter residue was then soaked in distilled water for $0.5 \mathrm{~h}$ and used the same method to extract and filter. The two filtrates were combined and concentrated to $168 \mathrm{~mL}$ using a vacuum rotary evaporator. Ultimately, Astragalus extract solution containing $1.07 \mathrm{~g} \mathrm{~mL}^{-1}$ of crude herb was obtained and the administration dosage for rats was $0.77 \mathrm{~mL} / 100 \mathrm{~g}$. Similarly, $34 \mathrm{~g}$ Leech were grounded into powder, and extracted and filtrated to $84 \mathrm{~mL}$ with the same methods as Astragalus. Finally, Leech extract solution containing $0.40 \mathrm{~g} \mathrm{~mL}^{-1}$ of crude herb was obtained and the administration dosage for rats was $0.38 \mathrm{~mL} / 100 \mathrm{~g}$. The above extraction methods and dosing volumes were based on ref. 37 . All these extracting solutions were stored at $4{ }^{\circ} \mathrm{C}$ until use.

\section{Systems pharmacology screening}

In order to further understand the mechanism of Astragalus and Leech on T2DN, the active compounds of these two Chinese herbs were screened by the method of systems pharmacology. TCMSP (http://lsp.nwu.edu.cn/tcmsp.php) is a unique systems pharmacology platform of Chinese herbal medicines that captures the relationships between drugs, targets and diseases. The database includes chemicals, targets and drug-target networks, and associated drug-target-disease networks, as well as pharmacokinetic properties for natural compounds involving oral bioavailability (OB), drug-likeness (DL), intestinal epithelial permeability, blood-brain-barrier (BBB), drug half-life (HL), aqueous solubility, etc. ${ }^{43}$ Because of the complexity of traditional Chinese medicines, it is impossible to verify so many compounds one by one, and the process (absorption, distribution, metabolism, and excretion, ADME) of compounds in the body is an important index of their druggability. Therefore, the use of ADME parameters as the initial screening of traditional Chinese medicine was conducive to rapid screening of active compounds. In general, compounds with $\mathrm{OB} \geqq 30 \%$, DL $\geqq$ 0.18 , BBB $>0.3$ and $\mathrm{HL} \geqq 4$ were selected as the active ingredient that can be absorbed by the body and may eventually have a therapeutic effect. ${ }^{\mathbf{3 9 4 - 4 6}}$ Since this database contains only the data of Astragalus, but there is no data of Leech. Accordingly, we determined the potential active compounds of Leech through literature search and the PubChem database. A myriad of literature studies have shown that the compounds existed in
Leech mainly contained hirudin, histamine, hirudinoidine A-C, cholesterol, campesterol, hexadecyl ethers of glycerol, nicotinic acid, uracil, uridine, succinic acid, hypoxanthine, inosine, xanthine, adenosine, phenylalanine, proline, alanine, valine, glycerin, palmiticacid, L-isoleucine. ${ }^{47-51}$ Meanwhile, we uploaded these compounds to the TCMSP database and searched for the corresponding ADME parameters. However, most of compounds in Leech have relatively small BBB and DL values. The compounds with $\mathrm{BBB}<-0.3$ were considered as nonpenetrating $(\mathrm{BBB}-)$, from -0.3 to +0.3 moderate penetrating $(\mathrm{BBB} \pm)$, and $>0.3$ strong penetrating $(\mathrm{BBB}+)$. Consequently, compounds of Leech with $\mathrm{OB} \geqq 30 \%$, DL $\geqq 0.01, \mathrm{BBB}>-0.3$ and $\mathrm{HL} \geqq 4$ were screened as potentially active compounds. Additionally, since TCMSP does not include ADME parameters for hirudin, [glycerol, 1-hexadecyl ether], palmiticacid, and hirudinoidine $\mathrm{A}-\mathrm{C}$, they were also considered as potential active compounds for Leech.

\section{Target prediction analysis}

All active compounds obtained from text digging and systems pharmacology analysis were uploaded to the PharmMapper server database (http://lilab.ecust.edu.cn/pharmmapper/ index.php) to predict and analyze the corresponding target proteins. PharmMapper server is an updated integrated pharmacophore matching platform with statistical method for potential target identification. ${ }^{52}$ Of the top 300 targets, $Z$-score $\geq 0.8$ was considered as criteria to screen potential target proteins. ${ }^{53,54}$

\section{KEGG pathway annotations}

UniProt numbers corresponding to all target proteins were uploaded into KEGG Mapper for pathway annotation. Search \& Color Pathway is an advanced version of the KEGG pathway mapping tool, where given objects (genes, proteins, compounds, glycans, reactions, drugs, etc.) are searched against KEGG pathway maps and found objects are marked in any background and foreground colors (bgcolor and fgcolor).

\section{Animal care and experiments}

Male Sprague-Dawley (SD) rats (weighing $135 \pm 15 \mathrm{~g}$ ) were obtained from the Experimental Animals Center of Guangdong Pharmaceutical University (License no. SCXK (Yue) 2013-0002) and kept in SPF grade experimental animal houses. All rats were allowed to have free access to food and pure water under standard animal conditions of temperature $\left(24 \pm 2{ }^{\circ} \mathrm{C}\right)$ and relative humidity ( $55 \pm 5 \%$ ), and a $12 \mathrm{~h}$ light/dark cycle. Animals were acclimatized to the new environment for one week prior to experimentation. After acclimatization, all rats were randomly divided into three groups of 10 rats each as follows: control group, T2DN group, and treated group. Control group' rats were given normal diet, while T2DN group and treated group' rats were fed with high-fat diet lasting for 4 weeks. After 4 weeks, all rats were fasted but have free access to water for $12 \mathrm{~h}$. T2DN group and treated group were randomly selected and injected intraperitoneally with STZ freshly prepared in citrate buffer (0.1 M, pH 4.5) at a single dosage of $40 \mathrm{mg} \mathrm{kg}^{-1}$ body weight, 
while control group was injected with the same volume of citrate buffer. On the $3^{\text {rd }}, 5^{\text {th }}, 7^{\text {th }}$ day after STZ injection, the fasting blood glucose (FBG) level of rats were measured daily by tail nick and glucometer after STZ injection, and rats with FBG > $16.70 \mathrm{mM}$ for three consecutive tests were defined as type 2 diabetic rats. ${ }^{11}$ Of 10 rats in the STZ treated group, 8 rats were scored as type 2 diabetic and all type 2 diabetes rats were fed with high-fat diet continuously to induce the occurrence of T2DN. Rats in model group with significantly higher blood glucose, UREA, and urinary protein creatinine ratios than control group were identified as T2DN rats. ${ }^{55}$ From the $8^{\text {th }}$ week onwards, the rats in treated group were given Astragalus and Leech extracting solutions once a day for 4 weeks in succession with the administration volume of Astragalus extract of $0.77 \mathrm{~mL} /$ $100 \mathrm{~g}$ and Leech extract of $0.38 \mathrm{~mL} / 100 \mathrm{~g}$. Control group and T2DN group were given the same volume of distilled water administration. The animal model and feeding methods of this experiment were referred to the relevant literatures. ${ }^{56-58}$ All rats among the control group, T2DN group and treated group were housed in metabolic cages (1 per cage) to collect fecal and urinary samples at the end of $12^{\text {th }}$ week. A whole night (from $20: 00$ p.m. to $8: 00$ a.m.), at least 3 pellets of feces from each rat in each group were collected, immediately frozen in liquid nitrogen and stored at $-80{ }^{\circ} \mathrm{C}$ until metabolomic analysis. ${ }^{59} 3$ days later, orbital venous blood was collected by capillary tube, and centrifuged at $4000 \mathrm{rpm}$ for $15 \mathrm{~min}$ after $30 \mathrm{~min}$ at room temperature. The supernatants were used to detect biochemical analysis. Fecal samples were stored at $-80{ }^{\circ} \mathrm{C}$ for NMR determination. Simultaneously, rats in three groups were sacrificed at the end of the $12^{\text {th }}$ week to remove the kidney tissues for pathological examination. Serum and kidney tissues were also kept in $-80{ }^{\circ} \mathrm{C}$ for subsequent analysis. All animal treatments were strictly in accordance with the National Institutes of Health Guide for the Care and Use of Laboratory Animals, and were approved by the Institutional Ethical Committee (IEC) of Guangdong Pharmaceutical University.

\section{Biochemical analysis and histopathology}

The levels of total cholesterol (TC), triglyceride (TG), high density lipoprotein (HDL), low density lipoprotein (LDL), urea (UREA), uric acid (UA) and creatinine (CREA) in serum, and $24 \mathrm{~h}$ urine protein excretion and creatinine in urine were carried out on an automatic biochemistry analyzer strictly according to the manufacturer's instructions on commercial kits. In addition, the body weight of the rats was also recorded and analyzed. The kidney tissues were fixed in 10\% formalin overnight, and then they were embedded in paraffin wax and $5 \mathrm{~mm}$ sections were prepared using a microtome. Tissue sections were stained with hematoxylin and eosin (HE) and examined by light microscopy.

\section{Sample preparations}

$250 \mathrm{mg}$ of feces and $1.0 \mathrm{~mL}$ PBS $\left(0.2 \mathrm{~mol} \mathrm{~L}{ }^{-1} \mathrm{Na}_{2} \mathrm{HPO}_{4} /\right.$ $\left.\mathrm{NaH}_{2} \mathrm{PO}_{4}, \mathrm{pH} 7.4\right)$ were added in $2.0 \mathrm{~mL}$ EP tube, and were vortexed for $30 \mathrm{~s}$. After that, the mixture was centrifuged (14 $000 \mathrm{rpm}, 10 \mathrm{~min}$ at $4{ }^{\circ} \mathrm{C}$ ) to remove any precipitates, and the supernatant was collected. $400 \mathrm{uL}$ of supernatant and $100 \mathrm{uL}$ of
TSP-containing $\mathrm{D}_{2} \mathrm{O}$ were added to a $5 \mathrm{~mm}$ NMR tube, mixed well and stored in a $4{ }^{\circ} \mathrm{C}$ refrigerator for further testing.

\section{${ }^{1} \mathrm{H}$ NMR data acquisition and processing}

${ }^{1} \mathrm{H}$ NMR spectra from feces were recorded using a Bruker Avance $500 \mathrm{MHz}$ spectrometer at $298 \mathrm{~K}$. Fecal extract samples were recorded using the water presaturated standard onedimensional NOESYPR1D pulse sequence (recycle delay- $90^{\circ}-t_{1}$ $90-t_{\mathrm{m}}-90^{\circ}$-acquisition) to obtain representative total metabolite compositions. 128 transients were collected into $32 \mathrm{k}$ data points using a spectral width of $10 \mathrm{kHz}$ with a relation delay of $3 \mathrm{~s}$, and the total echo time $(2 n \tau)$ was $100 \mathrm{~ms}$.

${ }^{1} \mathrm{H}$ NMR spectra were manually phased and baseline corrected, and spectral regions of $\delta 0.5-8.5 \mathrm{ppm}$ were then binned into contiguous segments of equal width $(0.02 \mathrm{ppm})$ by using AMIX software (V3.7.3, Bruker Biospin, Germany). The chemical shifts of the fecal spectra were referenced to the TSP signal at $\delta 0.00$. The integral values at $\delta 4.7-5.2$ were set to be zero in order to eliminate the effects of water. Finally, the remaining spectral segments from the region $\delta 0.5-8.5$ were normalized to the total sum of the spectral integrals in order to avoid any significant concentration differences. The resulting normalized integral data were then subjected to multivariate data analysis and biomarker identification using SIMCA 13.0 software (Umetrics, Sweden).

\section{Multivariate data analysis and biomarker identification}

Principal component analysis (PCA), an unsupervised method, was initially performed to the unit variance (UV)-scaled ${ }^{1} \mathrm{H}$ NMR data to generate an overview of inherent clustering. Whereafter, partial least squares discriminant analysis (PLS-DA) and orthogonal projection to latent structure discriminant analysis (OPLS-DA), supervised methods, were constructed using pareto scaling to maximize the distinction between two groups. Loading plot from PCA model was used to highlight the spectral regions (total variations) strongly contributing to each component. PLS-DA model was validated with a permutation test (200 permutations). OPLS-DA was validated by both a 7 -fold crossvalidation and ANOVA of the cross-validated residuals (CVANOVA) methods. ${ }^{60}$ Potential biomarkers were extracted from $S$-plots based on their contribution to the variation. Eventually, VIP $>1$ and $P$ value $<0.05$ were selected as the potential biomarkers.

\section{Correlation analysis of biomarkers and biochemical parameters}

In order to further probe the potential relationship between biomarkers and biochemical parameters, Pearson's correlation analysis was performed on these 22 biomarkers and 9 biochemical parameters. Positive values masked in the correlation diagram were shown with red color and negative values were indicated with blue color. Besides, the correlation coefficient and color can reflect the strength of the relationship. Significantly correlated at $* P<0.05, * * P<0.01$ (two-sided test). 


\section{Construction of metabolic pathways}

MetaboAnalyst (http:/www.metaboanalyst.ca), an integrated web-based platform for comprehensive analysis and interpretation of metabolomic data, handles most of the common metabolomic data types from most kinds of metabolomics platforms (e.g. NMR) for most kinds of metabolomics experiments (e.g. targeted, untargeted, quantitative). ${ }^{61}$ Thus, all potential metabolites selected from above were uploaded and analyzed via metabolite set enrichment analysis and pathways analysis in MetaboAnalyst. The specified pathway analysis algorithms were: hypergeometric test was used to over representation analysis, and relative-between centrality was applied for pathway topology analysis. Simultaneously, False Discovery Rate (FDR), a correction for multiple comparisons, was also devoted to indicate statistical significance in order to protect against type I error rate inflation. The pathways' $P$ values $(P<$ 0.05 ) were regarded as potential therapy pathways in T2DN model treated by Astragalus and Leech.

\section{Analysis by combining systems pharmacology and fecal metabonomics}

$P<0.05$ pathways screened from fecal metabonomics was mapped to all pathways selected by systems pharmacology to look for overlapping pathways. Overlapping pathways were considered as potential pathway for the treatment of T2DN with Astragalus and Leech. After that, target proteins corresponding to these pathways were searched by KEGG. These target proteins were reversely used to seek the corresponding compounds in Astragalus and Leech. Taken together, an interaction network of herb-active compound-target protein-pathway for the improvement of T2DN by Astragalus and Leech was established.

\section{Statistical analysis}

Metabolites and biochemical data were analyzed by using SPSS software (version 20.0, IBM Inc., USA) with Student's $t$ test or one-way analysis of variance (ANOVA) and Bonferroni multiple comparisons, and statistically significant difference was defined as $P<0.05$. Additionally, the biomarkers (VIP $>1$ and $P<$ 0.05 ) were assessed by multivariate statistical analysis.

\section{Results}

\section{Biochemical parameters and histopathological analysis}

Biochemical parameters determined by biochemical analysis and representative images of the histopathological examination of HE staining sections of kidney tissues from three groups are shown in Fig. 1. It was found that TC, TG, LDL, UREA and $24 \mathrm{~h}$ urine protein in T2DN group all increased significantly compared with those in control group, while HDL and weight decreased significantly. This indicated that significant impaired renal function occurred in T2DN rats. However, TC, TG, LDL, UREA and $24 \mathrm{~h}$ urine protein decreased after treatment with Astragalus and Leech, while HDL, UA and weight were found to increase. This indicated that Astragalus and Leech have a therapeutic effect on T2DN. HE staining results showed that there was no histological change in the kidneys of the control rats, while the pathological changes of the kidney of the T2DN rats showed a series of typical pathological changes of renal injury, including glomerular enlargement, mesangial expansion, glomerular basement membrane (GBM) thickening, glomerular atrophy and tubular epithelial cell vacuolar degeneration (corresponding to the numbers 1-5 in HE staining of Fig. 1, respectively). Compared with the T2DN group, these pathological abnormalities improved to some extent after treatment with Astragalus and Leech, and were close to control group. Combined with data of biochemical parameters and histopathological analysis, our results demonstrated that the T2DN model was well-established in this study. Meanwhile, this also showed that Astragalus and Leech have a certain therapeutic effect on T2DN.

\section{Active compound screening}

Finally, 4 potentially active compounds were screened from Astragalus, including hederagenin; $(3 S, 8 S, 9 S, 10 R, 13 R, 14 S, 17 R)$ 10,13-dimethyl-17-[(2R,5S)-5-propan-2-yl-octan-2-yl]-2,3,4,7,8,9, $11,12,14,15,16,17$-dodecahydro- $1 H$-cyclopenta $[a]$ phenanthren3-ol; 3,9-di-O-methylnissolin; $\quad(6 a R, 11 a R)-9,10$-dimethoxy6a,11a-dihydro-6H-benzofurano[3,2-c]chromen-3-ol; 16 potentially active compounds were screened from Leech, including hirudin; histamine; campesterol; hypoxanthine; [glycerol, 1hexadecyl ether]; L-isoleucine; cholesterol; nicotinic acid; uracil; phenylalanine; valine; palmiticacid; proline; hirudinoidine A-C (Table S1, ESI $\dagger$ ). The structural of these compounds were uploaded into the PharmMapper database to predict the corresponding targets.

\section{Target prediction result}

Target prediction results showed that 310 target proteins met the screening criteria and were likely to be affected by the active compounds (Table S2, ESI $\dagger$ ). Therefore, we imported these target proteins into the KEGG database for annotation and searched for the corresponding pathways.

\section{Target corresponding pathway}

The results of the KEGG pathway annotation showed that 310 target proteins may be affected by 210 pathways. These pathways included MAPK signaling pathway, Ras signaling pathway, purine metabolism and pyruvate metabolism, etc. All target proteins' corresponding pathways predicted by KEGG were available in ESI, Table S3.†

\section{${ }^{1} \mathrm{H}$ NMR spectra of fecal sample}

The representative ${ }^{1} \mathrm{H}$ NMR NOESYPR1D spectra of fecal samples from control, T2DN and treated groups were shown in Fig. 2. The peaks in the spectra were assigned using previously reported ${ }^{1} \mathrm{H}$ NMR assignments for metabolites, which were obtained from HMDB database and literatures. ${ }^{\mathbf{6 0 , 6 2 - 6 5}}$ As a result, 31 endogenous metabolites were assigned. 

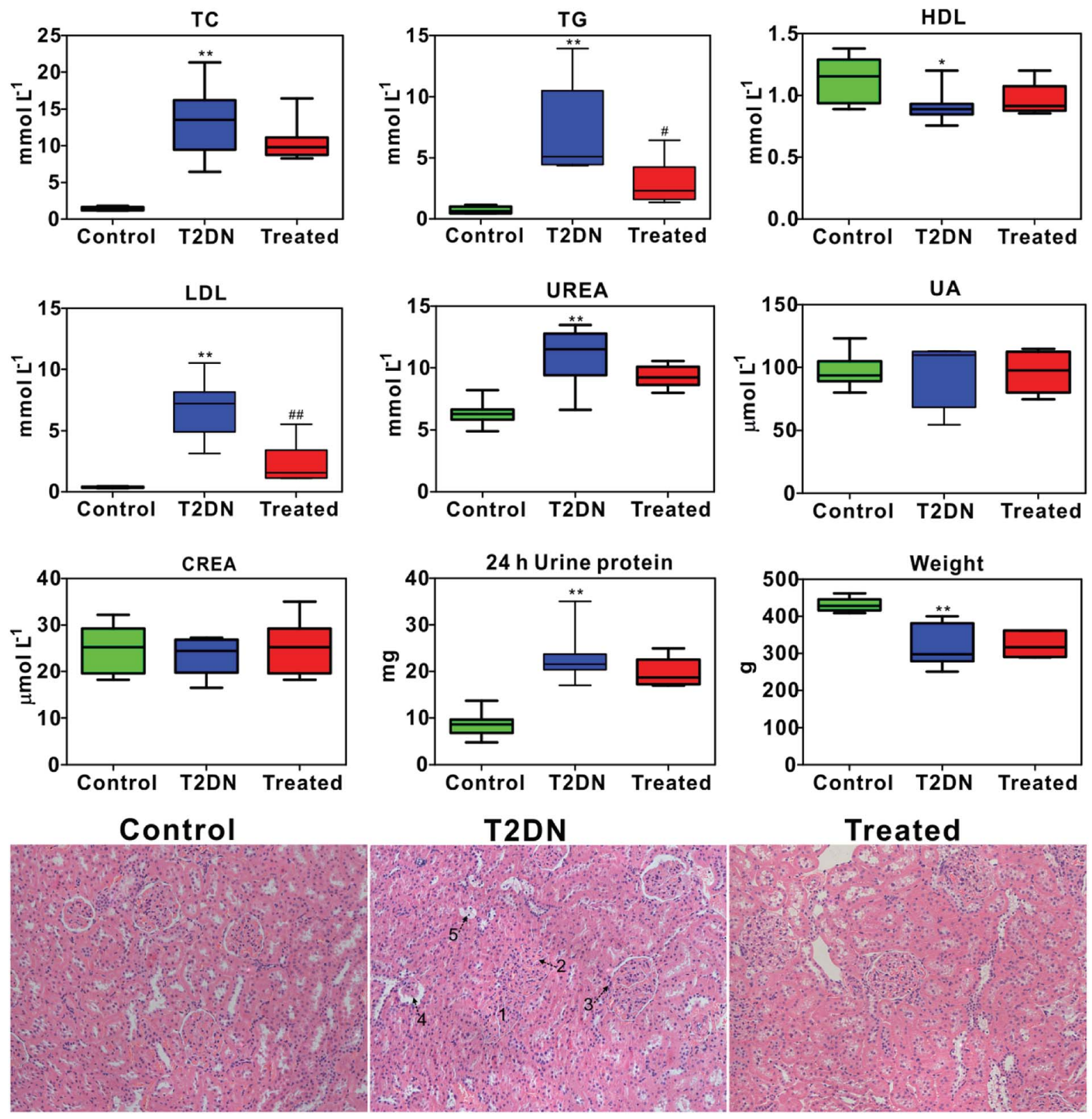

Fig. 1 Box plots of concentration of biochemical and weight data from different groups of rats (above); * as compared with the control group, * $P$ $<0.05,{ }^{* * P}<0.01$; ${ }^{*}$ as compared with the T2DN group, ${ }^{\#} P<0.05,{ }^{\# \#} P<0.01$ (one-way ANOVA with a Bonferroni correction). Representative HE staining (magnification 20x) of kidney tissues among control, T2DN and treated group (below).

\section{Pattern recognition analysis and identification of biomarker}

Fig. 3A1 and A2 showed a PCA and 3D-PCA score plot of feces from control group, T2DN group and treated group. As seen in the figure, control group, T2DN group and treated group manifested a better separation, indicating that there were significant differences among three groups. Loading plot (Fig. S1, ESI $\dagger$ ) from PCA model was also used to highlight the spectral regions (total variations) strongly contributing to each component. The validation plot permutation tests showed that the PLS-DA model built for three groups was robust and credible (Fig. S2, ESI $\dagger$ ). Therefore, OPLS-DA scores plots (Fig. 3B1 and C1, respectively) were performed to maximize the distinction between groups, and $S$-plots (Fig. 3B2 and C2, respectively) were utilized for identification of potential biomarkers obtained from two groups. The CV-ANOVA was used to validate the OPLSDA models, and the corresponding $p$-values were calculated, which indicated that the OPLS-DA models for feces were valid. For $S$-plots, the further the variable deviates from the origin, the higher the value of Variable Importance in the Projection (VIP) will be obtained. Therefore, those variables farthest from the origin in the $S$-plot were regarded as biomarkers. According to the VIP values (VIP $>1)$ and $P$ values $(P<0.05)$ from OPLS-DA scores plots and $S$-plots, 22 discriminatory metabolites among groups were finally screened out and selected as the potential biomarkers (Table 1, Table S5, ESI $\dagger$ ). 


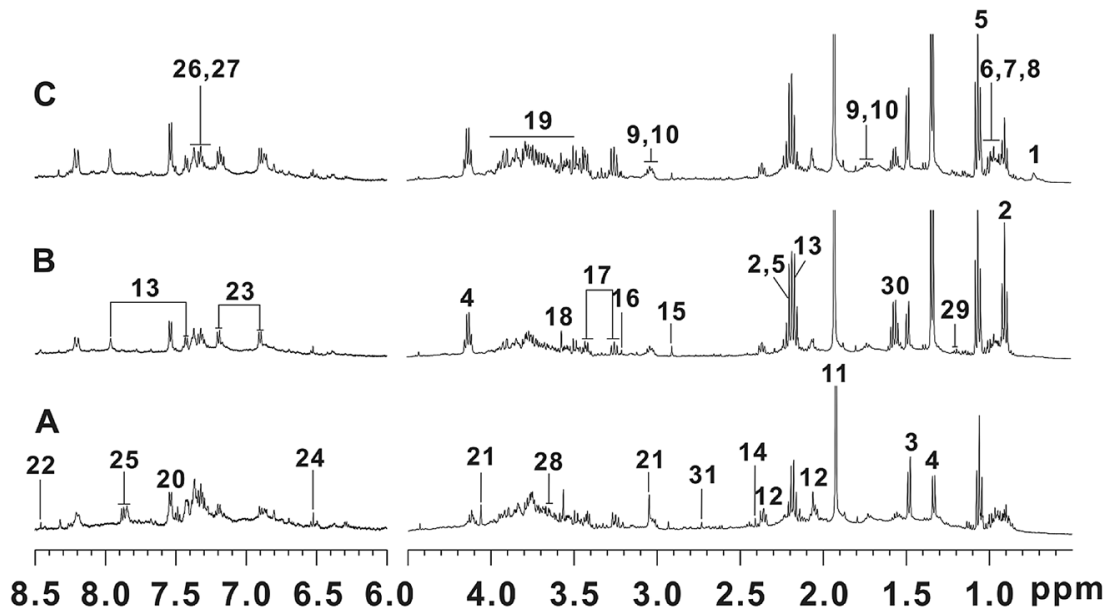

Fig. 2 Typical $500 \mathrm{MHz}{ }^{1} \mathrm{H}$ NMR spectra of fecal extracts: (A) control group; (B) T2DN group; (C) treated group. Keys: (1) deoxycholate; (2) $n$ butyrate; (3) alanine; (4) lactate; (5) propionate; (6) valine; (7) leucine; (8) isoleucine; (9) lysine; (10) cadaverine; (11) acetate; (12) glutamate; (13) $N$ acetyl-5-aminosalicylate; (14) succinate; (15) trimethylamine; (16) choline; (17) taurine; (18) glycine; (19) $\alpha$-glucose/ $\beta$-glucose; (20) tryptophan; (21) creatinine; (22) formate; (23) tyrosine; (24) fumarate; (25) benzoic acid; (26) phenylalanine; (27) phenylacetic acid; (28) glycerol; (29) 3hydrobutyrate; (30) citrulline; (31) dimethylamine.

\section{Correlation analysis of biomarkers and biochemical parameters}

Biomarkers and biochemical parameters $(|r| \geq 0.707, P<0.05)$ between control group and treated group were significantly correlated, while there was no significant correlation in T2DN group (Fig. 4). In control group, X2 [deoxycholate] was positively related to Y6 [UA]; X3 [leucine] was negatively related to Y2 [TG] and Y9 [24 h urine protein]; X4 [lysine] was negatively related to Y9 [24 h urine protein]; X8 [lactate] was positively related to Y2 [TG]; X14 [succinate] was negatively correlated to Y7 [CREA]; X15 [dimethylamine] was negatively correlated to Y9 [24 h urine protein]; X16 [trimethylamine] was negatively related to $\mathrm{Y} 7$ [CREA]; X18 [phenylalanine] was negatively related to Y9 [24 h urine protein]; X20 [a-glucose] was negatively related to Y2 [TG]; $\mathrm{X} 21$ [glycerol] was negatively related to Y2 [TG]; X22 [taurine] was negatively related to Y2 [TG]). In treated group, however, X6 [3-hydroxybutyrate] was positively correlated to Y2 [TG] and Y7 [CREA], and negatively related to $\mathrm{Y} 9$ [24 $\mathrm{h}$ urine protein]; X7 [isoleucine] was negatively related to Y5 [UREA]; X8 [lactate] was positively related to $\mathrm{Y} 2$ [TG], Y4 [LDL] and Y7 [CREA]; X12 [acetate] was positively correlated with Y1 [TC]. This was consistent with the results of Zhang et al. ${ }^{\mathbf{6 6}}$

We believed that this might be due to the occurrence of T2DN in rats, thereby leading to metabolic disorders in vivo of rats, and the original correlation and dynamic balance between metabolites and biochemical parameters were broken. In addition, we could also clearly find from Fig. 4 that the number of significant correlation between metabolites and biochemical parameters in treated group (8) was obviously close to that in control group (12), while there was no significant correlation between metabolites and biochemical parameters in T2DN group. Meanwhile, the correlation between all metabolites and biochemical parameters in treated group was closer to control group than that in T2DN group. Thus, it could be seen that after treatment with Astragalus and Leech, to some extent, the metabolism of T2DN rats was effectively regulated, which partially restored the correlation between metabolites and biochemical parameters. This also showed that Astragalus and Leech did have a certain therapeutic effect on T2DN.

\section{Pathways analysis}

The metabolome view in Fig. 5 showed all matched pathways according to the $p$ values from the pathway enrichment analysis and pathway impact values (from 0 to 1 ) from the pathway topology analysis. 22 metabolites were used to metabolite set enrichment analysis and pathway analysis using MetaboAnalyst tool (Fig. 5A and B, respectively). The results showed that 22 metabolites corresponded to 23 pathways, of which 6 pathways were $P<0.05$, including: (1) aminoacyl-tRNA biosynthesis; (2) valine, leucine and isoleucine biosynthesis; (3) butanoate metabolism; (4) valine, leucine and isoleucine degradation; (5) propanoate metabolism; (6) pyruvate metabolism. Labels in Fig. 5B correspond to numbering of pathways in Table 2.

\section{Correlation analysis of biomarkers and pathways}

The correlation between biomarkers and pathways was constructed according to the KEGG database and literatures. It could be clearly seen from Fig. 6 that metabolites, target proteins and pathways in T2DN rats have occurred a series of metabolic disorders. After the treatment of Astragalus and Leech, however, the corresponding metabolite levels have been restored. This also showed that Astragalus and Leech, to some extent, could improve the metabolic disturbance of T2DN rats in vivo.

\section{Combined with systems pharmacology and fecal metabonomics analysis}

$P<0.05$ pathways screened from fecal metabonomics was mapped to all pathways selected by systems pharmacology to look for 

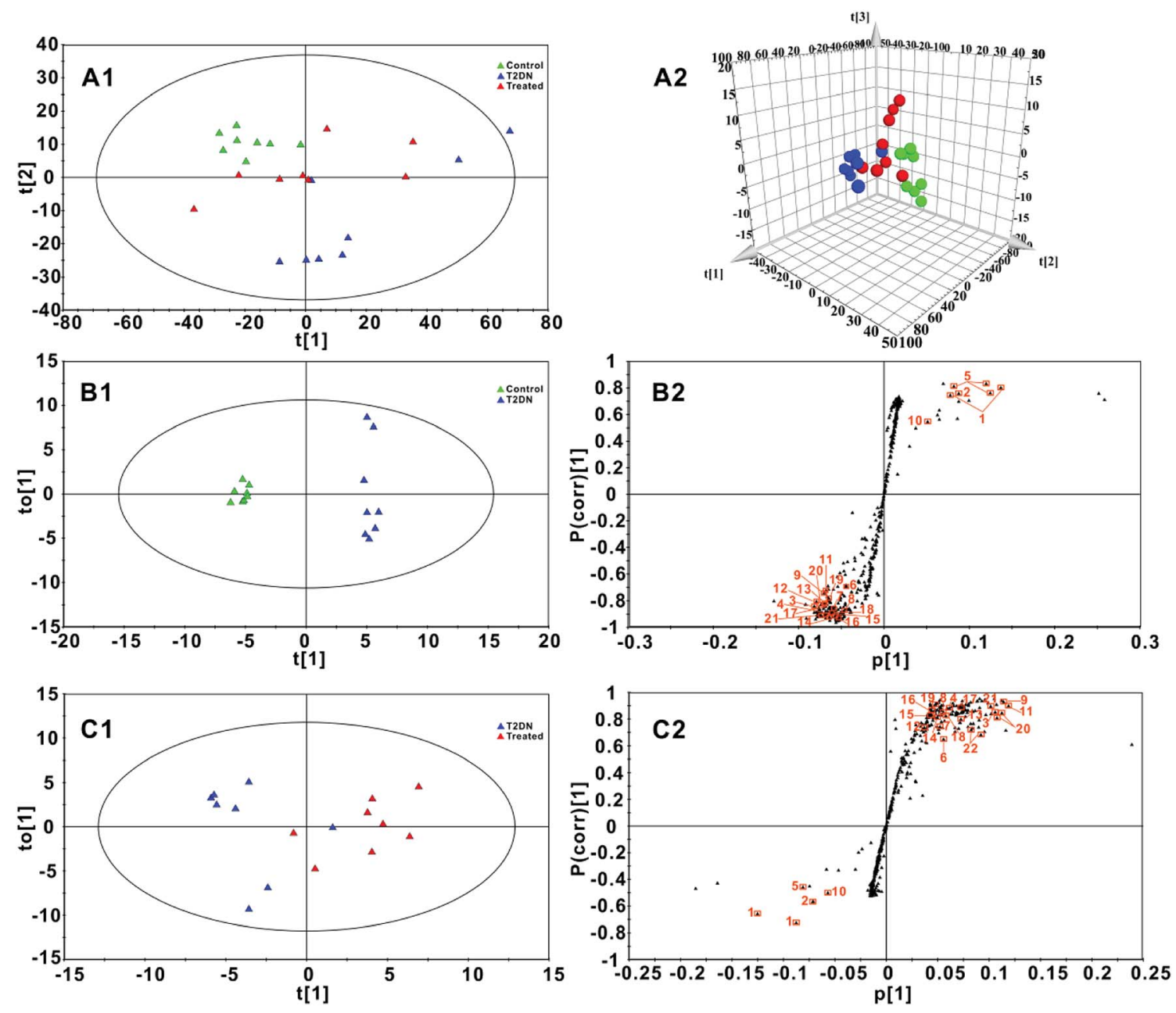

Fig. 3 (A1 and A2): PCA score plot and 3D-PCA score plot for three groups $\left(R^{2} X=0.963, Q^{2}=0.941\right)$ based on ${ }^{1} H$ NMR spectra of fecal samples; (B1 and B2): OPLS-DA score plot and S-plot for control group and T2DN group $\left(R^{2} X=0.932, R^{2} Y=0.993, Q^{2}=0.970, p\right.$-value $\left.=0.0001\right) ;(C 1$ and C2): OPLS-DA score plot and S-plot for T2DN group and treated group $\left(R^{2} X=0.757, R^{2} Y=0.703, Q^{2}=0.581, p\right.$-value $\left.=0.0352\right)$. Control group ( $\boldsymbol{\Lambda}$, green), T2DN group ( $\boldsymbol{\Lambda}$, blue) and treated group ( $\boldsymbol{\Delta}$, red). The axes plotted in the $S$-plots from the predictive component are p[1] vs $p$ (corr)[1], representing the magnitude and reliability, respectively. Variables labeled with orange squares ( $\square$ ) could be exploited as potential biomarkers and the labels next to the orange squares correspond to the numbers of metabolites in Table 1.

overlapping pathways. The mapping results showed that there were 6 overlapping pathways. They were (1) aminoacyl-tRNA biosynthesis; (2) valine, leucine and isoleucine biosynthesis; (3) butanoate metabolism; (4) valine, leucine and isoleucine degradation; (5) propanoate metabolism; (6) pyruvate metabolism. Combining with systems pharmacology and fecal metabonomics, an interaction network of herb-active compound-target proteinpathway regulated by Astragalus and Leech was established basically (Fig. 7). As shown in Fig. 7, the same active compounds in Astragalus and Leech could act on multiple target proteins simultaneously, thereby affecting the same or different pathways. Different active compounds could also act on the same target protein or different target proteins, thus affecting the same pathways. To a certain extent, this was consistent with the overall synergistic mechanism of multi-component, multi-target and multi-pathway of TCM. Besides, hederagenin and $(3 S, 8 S, 9 S, 10 R, 13 R, 14 S, 17 R)-10,13-d i m e t h y l-17-[(2 R, 5 S)-5$-propan-2-yl- octan-2-yl]-2,3,4,7,8,9,11,12,14,15,16,17-dodecahydro- $1 H$-cyclopenta [a]phenanthren-3-ol had not their corresponding target proteins and pathways. Corresponding targets and pathways of the 9 compounds in Leech also could not be found, including: histamine; campesterol; hypoxanthine; [glycerol, 1-hexadecyl ether]; cholesterol; nicotinic acid; uracil; palmiticacid and proline. So they did not appear in Fig. 7. At last, an interactive network map was constructed by the remaining 2 active compounds of Astragalus and 7 active compounds of Leech, and their corresponding targets and pathways.

\section{Discussion}

Metabonomics, with lots of advantages, including short analysis time, rich structure information, nondestructive analysis and relatively little sample, has already become a powerful tool for understanding the therapeutic mechanisms of TCM 
Table 1 Statistical analysis results of the main metabolite in feces ${ }^{a}$

\begin{tabular}{|c|c|c|c|c|c|c|}
\hline No. & Metabolites & Chemical shift (ppm) & T2DN group & Treated group & $\begin{array}{l}\text { VIP values in } \\
\text { T2DN group }\end{array}$ & $\begin{array}{l}\text { VIP values in treated } \\
\text { group }\end{array}$ \\
\hline 1 & $n$-Butyrate & $0.90(\mathrm{t}), 1.56(\mathrm{~m}), 2.16(\mathrm{t})$ & $\uparrow * *$ & $\downarrow^{\#}$ & $2.43,4.34$ & $4.71,2.00$ \\
\hline 3 & Leucine & $0.96(\mathrm{~d}), 0.97(\mathrm{~d}), 1.70(\mathrm{~m}), 1.72(\mathrm{~m}), 3.74(\mathrm{~m})$ & $\downarrow * *$ & $\uparrow^{\# \#}$ & $2.43,2.63$ & $2.51,2.96$ \\
\hline 4 & Valine & $0.99(\mathrm{~d}), 1.05(\mathrm{~d}), 2.29(\mathrm{~m}), 3.62(\mathrm{~d})$ & $\downarrow * *$ & $\uparrow^{\# \#}$ & 2.08 & 1.99 \\
\hline 5 & Propionate & $1.06(\mathrm{t}), 2.19(\mathrm{q})$ & $\uparrow * *$ & $\downarrow$ & $3.78,3.95$ & 3.00 \\
\hline 8 & Lactate & $1.33(\mathrm{~d}), 4.11(\mathrm{q})$ & $\downarrow * *$ & $\uparrow^{\#}$ & 1.46 & $1.75,1.46$ \\
\hline 9 & Alanine & $1.48(\mathrm{~d}), 3.78(\mathrm{q})$ & $\downarrow * *$ & $\uparrow^{\# \#}$ & $2.23,2.18$ & $2.96,3.36$ \\
\hline 10 & Citrulline & $1.57(\mathrm{~m})$ & $\uparrow *$ & $\downarrow$ & 1.64 & 2.15 \\
\hline 11 & Lysine & $1.48(\mathrm{~m}), 1.73(\mathrm{~m}), 1.91(\mathrm{~m}), 3.03(\mathrm{t}), 3.77(\mathrm{t})$ & $\downarrow * *$ & $\uparrow^{\# \#}$ & $1.88,2.94$ & $2.03,3.36$ \\
\hline 12 & Acetate & $1.91(\mathrm{~s})$ & $\downarrow * *$ & $\uparrow^{\#}$ & 2.45 & 2.34 \\
\hline 17 & Creatinine & $3.05(\mathrm{~s}), 4.06(\mathrm{~s})$ & $\downarrow * *$ & $\uparrow^{\# \#}$ & 2.26 & 2.25 \\
\hline 18 & Phenylalanine & $\begin{array}{l}\text { 7.44(m), 7.39(m), 7.33(m), 3.17(dd), } \\
3.30(\mathrm{dd}), 3.99(\mathrm{dd})\end{array}$ & $\downarrow * *$ & $\uparrow=\#$ & $1.72,1.92$ & $1.67,1.82$ \\
\hline 19 & Tryptophan & $\begin{array}{l}3.31(\mathrm{dd}), 3.50(\mathrm{dd}), 4.07(\mathrm{dd}), 7.33(\mathrm{~s}) \\
7.55(\mathrm{~d})\end{array}$ & $\downarrow^{* *}$ & $\uparrow^{\# \#}$ & 2.05 & 1.76 \\
\hline 20 & $\alpha$-Glucose & $\begin{array}{l}3.42(\mathrm{t}), 3.53(\mathrm{dd}), 3.71(\mathrm{t}), 3.73(\mathrm{dd}) \\
3.83(\mathrm{~m}), 3.84(\mathrm{dd}), 5.249(\mathrm{~d})\end{array}$ & $\downarrow^{* *}$ & $\uparrow^{\# \#}$ & $2.35,2.09$ & $2.93,2.95$ \\
\hline 21 & Glycerol & $3.65(\mathrm{dd})$ & $\downarrow * *$ & $\uparrow^{\# \#}$ & 2.34 & 3.02 \\
\hline 22 & Taurine & $3.27(\mathrm{t}), 3.43(\mathrm{t})$ & $\downarrow$ & $\uparrow^{\#}$ & - & $2.25,2.35$ \\
\hline
\end{tabular}

formulas. In present study, fecal metabonomics is used to analyze endogenic metabolites of T2DN rats and T2DN rats treated by Astragalus and Leech. 22 metabolic markers are finally found, mainly involving amino acid metabolism (leucine, valine, isoleucine, alanine, lysine, glutamate, taurine, phenylalanine, tryptophan), energy metabolism (lactate, succinate, creatinine, $\alpha$-glucose, glycerol), ketone body and fatty acid metabolism (3-hydroxybutyrate, acetate, $n$-butyrate, propionate), methylamine metabolism (dimethylamine, trimethylamine), and secondary bile acid metabolism and urea cycle (deoxycholate, citrulline). Based on the analysis of Astragalus and Leech by combining with systems pharmacology, it is found that there are 6 pathways that are closely related to the improvement of T2DN, including (1) aminoacyl-tRNA biosynthesis; (2) valine, leucine and isoleucine biosynthesis; (3) butanoate metabolism; (4) valine, leucine and isoleucine degradation; (5) propanoate metabolism; (6) pyruvate metabolism. Comparing with the differential metabolites of other biosamples (i.e. serum, urine, kidney extracts) from T2DN model analyzed by metabolomics technique, it was found that of the 22 metabolites in our study, 6 metabolites ( $n$-butyrate, deoxycholate, propionate, tryptophan, taurine and glycerol) associated with T2DN were newly discovered in fecal samples, while 16 metabolites (leucine, valine, 3-hydroxybutyrate, isoleucine, lactate, alanine, citrulline, lysine, acetate, glutamate, succinate, dimethylamine, trimethylamine, creatinine, phenylalanine and $\alpha$-glucose) have been found in other biological samples. ${ }^{\mathbf{1 1 , 1 2 , 6 7 - 7 0}}$ These 6 metabolites were mainly derived from the intestinal flora, and related to amino acid metabolism, fatty acid metabolism, and secondary bile acid metabolism.

\section{Amino acid metabolism}

As can be seen from Table 1, 9 kinds of amino acids in T2DN group are significantly decreased, while increase significantly in treated group. This shows that amino acid metabolism in T2DN rats is obviously disturbed. A large number of amino acids (e.g. valine, isoleucine, phenylalanine, tryptophan, alanine and glutamate) can be converted into glucose by gluconeogenesis, leading to hyperglycemia eventually in T2DN rats. Leucine, isoleucine and valine are branched chain amino acids (BCAAs) among 8 kinds of essential amino acids for humans. Valine, leucine and isoleucine biosynthesis/degradation are two important pathways BCAAs participate in. Leucine plays crucial roles in the determination of globular protein structures and the interaction of the transmembrane domains of membranous proteins with phospholipid bilayers. ${ }^{71}$ BCAAs are involved in immunity, the therapy of metabolic disorders, and the induction of antioxidant DNA repair. ${ }^{72,73}$ Meanwhile, they also have been shown to affect protein metabolism, apoptosis and regeneration of hepatocytes, and insulin resistance. ${ }^{74}$ Screening 


\section{A Control}

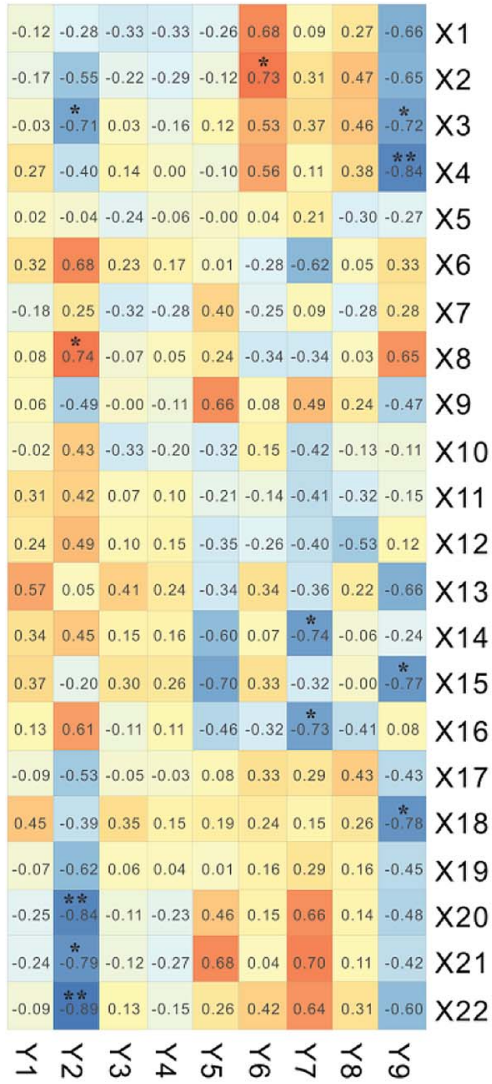

B T2DN

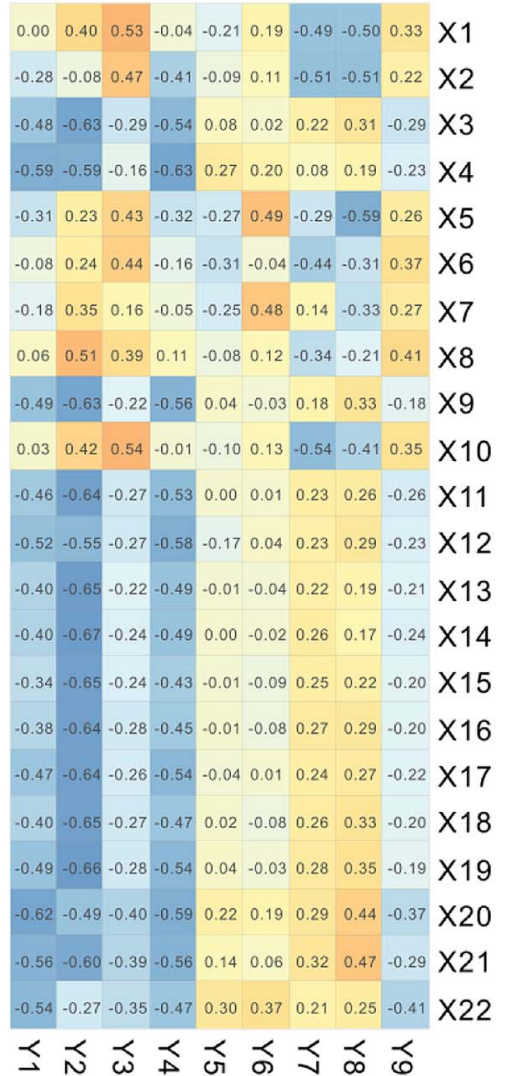

\section{Treated}

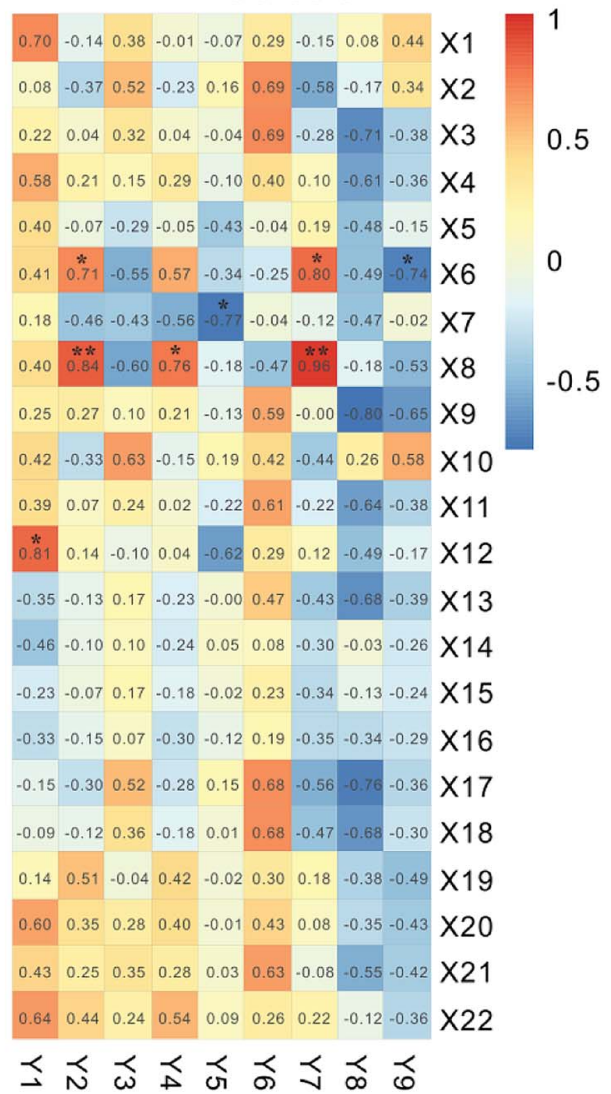

Fig. 4 Pearson's correlation analysis of 22 biomarkers and 9 biochemical parameters in control, T2DN and treated groups, respectively. X1-X22 correspond to numbering of metabolites in Table 1. Y1-Y9 represent TC, TG, HDL, LDL, UREA, UA CREA, weight, and 24 h urine protein, respectively. Significantly correlated at $* P<0.05, * * P<0.01$ (two-sided test).

\section{A Metabolite Sets Enrichment Overview}

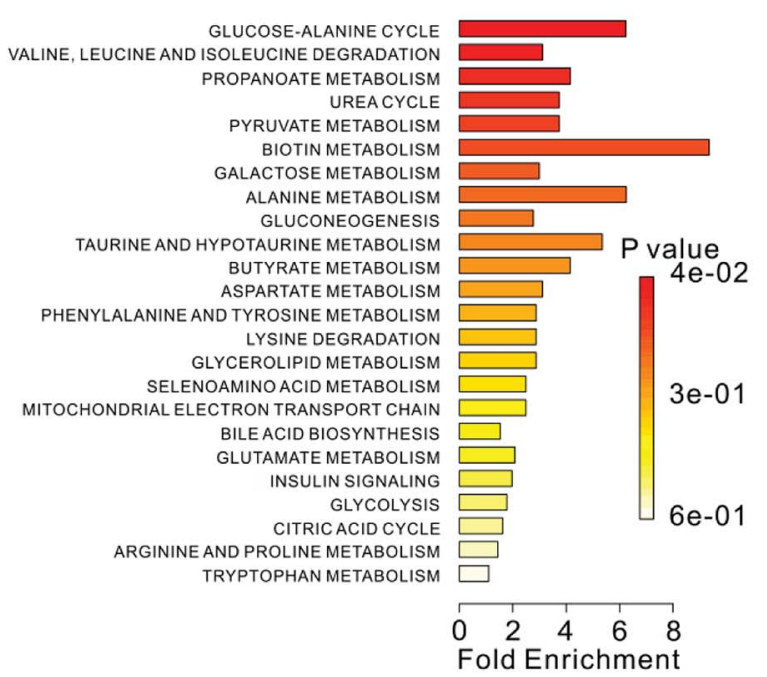

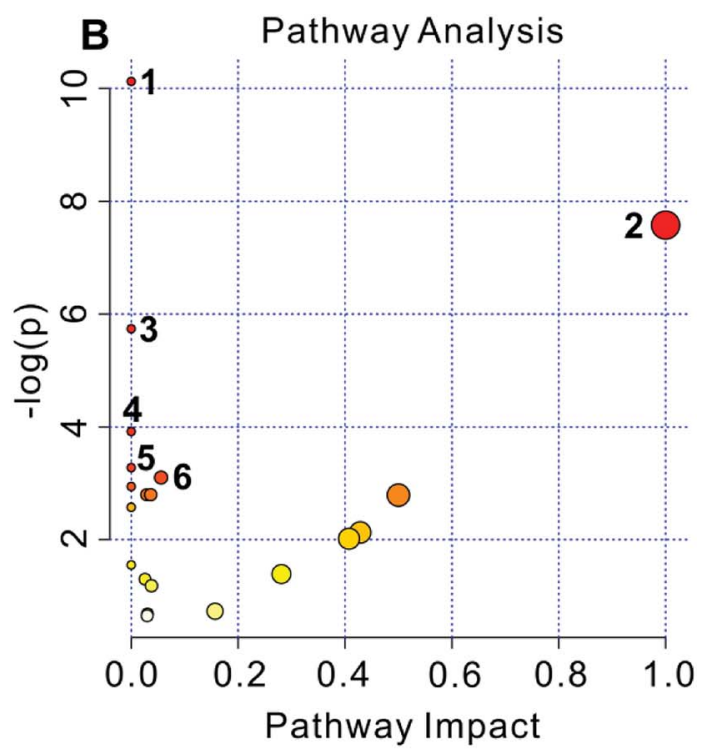

Fig. 5 The metabonome view above shows all matched pathways according to $p$ values from pathway enrichment analysis (A) and pathway impact values from pathway topology analysis (B). The bubble size is proportional to the impact of each pathway and the bubble color denotes the significance, from highest in red to lowest in white. Labels correspond to numbering of pathways in Table 2. $P$ values and pathway impact values (from 0 to 1 ) are corresponding to " $P$-value" and "Impact" column in Table 2, respectively. 
Table 2 Results of pathway analysis using MetaboAnalyst ${ }^{a}$

\begin{tabular}{|c|c|c|c|c|c|c|c|}
\hline No. & Pathway name & Hits & Total & $P$-value & $-\log (p)$ & FDR & Impact \\
\hline 1 & Aminoacyl-tRNA biosynthesis & 7 & 67 & $4.0088 \times 10^{-5}$ & 10.124 & 0.0032471 & 0.0 \\
\hline 2 & Valine, leucine and isoleucine biosynthesis & 3 & 11 & $5.1084 \times 10^{-4}$ & 7.5795 & 0.020689 & 0.99999 \\
\hline 4 & Valine, leucine and isoleucine degradation & 3 & 38 & 0.019827 & 3.9207 & 0.40151 & 0.0 \\
\hline 5 & Propanoate metabolism & 2 & 20 & 0.037657 & 3.2792 & 0.47441 & 0.0 \\
\hline 6 & Pyruvate metabolism & 2 & 22 & 0.044926 & 3.1027 & 0.47441 & 0.05583 \\
\hline 9 & Galactose metabolism & 2 & 26 & 0.060871 & 2.799 & 0.47441 & 0.03644 \\
\hline 10 & Phenylalanine, tyrosine and tryptophan biosynthesis & 1 & 4 & 0.06137 & 2.7908 & 0.47441 & 0.5 \\
\hline 11 & Synthesis and degradation of ketone bodies & 1 & 5 & 0.076141 & 2.5752 & 0.47441 & 0.0 \\
\hline 12 & D-Glutamine and D-glutamate metabolism & 1 & 5 & 0.076141 & 2.5752 & 0.47441 & 0.0 \\
\hline 13 & Biotin metabolism & 1 & 5 & 0.076141 & 2.5752 & 0.47441 & 0.0 \\
\hline 14 & Taurine and hypotaurine metabolism & 1 & 8 & 0.11913 & 2.1275 & 0.68928 & 0.42857 \\
\hline 19 & Citrate cycle (TCA cycle) & 1 & 20 & 0.27277 & 1.2991 & 1.0 & 0.02566 \\
\hline 20 & Starch and sucrose metabolism & 1 & 23 & 0.30697 & 1.181 & 1.0 & 0.03778 \\
\hline 21 & Tryptophan metabolism & 1 & 41 & 0.4821 & 0.7296 & 1.0 & 0.15684 \\
\hline 22 & Arginine and proline metabolism & 1 & 44 & 0.50683 & 0.67958 & 1.0 & 0.03042 \\
\hline 23 & Primary bile acid biosynthesis & 1 & 46 & 0.52269 & 0.64877 & 1.0 & 0.03042 \\
\hline
\end{tabular}

${ }^{a}$ The "Total" is the number of compounds in the pathway; the "Hits" represents the actual matched number from the user uploaded data.

results of systems pharmacology show that 3,9-di-O-methylnissolin in Astragalus, and hirudin, L-isoleucine and valine in Leech can act on various target proteins (i.e. $\mathrm{L}$-serine dehydratase [EC: 4.3.1.19], branched-chain amino acid aminotransferase [EC: 2.6.1.42], 3-hydroxyacyl-CoA dehydrogenase [EC: 1.1.1.35], isovaleryl-CoA dehydrogenase [EC: 1.3.8.4], and

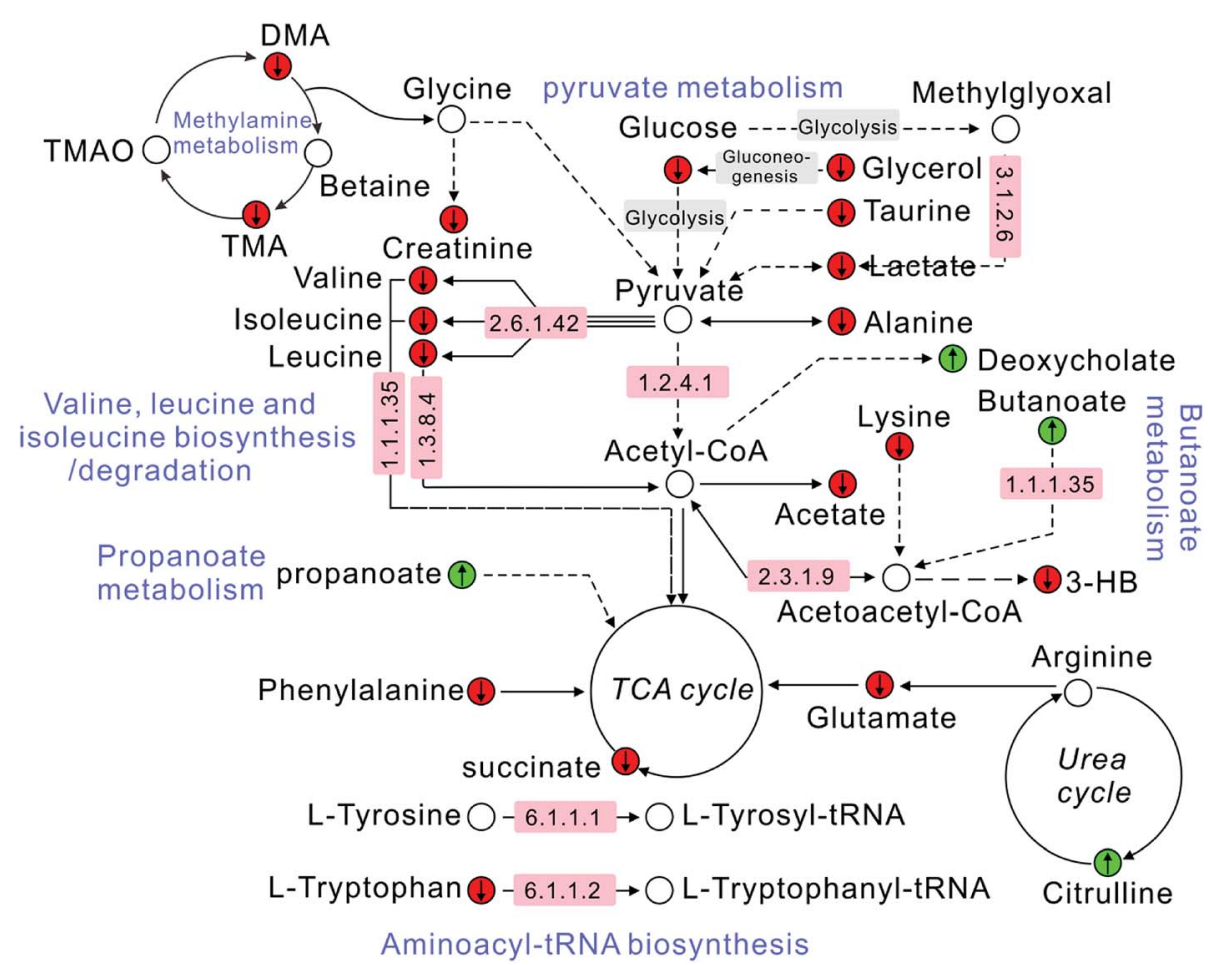

Fig. 6 Schematic diagram of the metabolic pathway alterations among the biomarkers associated with T2DN according to the KEGG database. " $\uparrow$ " represents a significant elevation in metabolites in T2DN group, while " $\downarrow$ " indicates a significant reduction. A red color (๑) indicates increased metabolites in treated group, while a green color (๑) shows decreased metabolites. The number in the pink region corresponds to the ID of target proteins in ESI, Table S4.† 


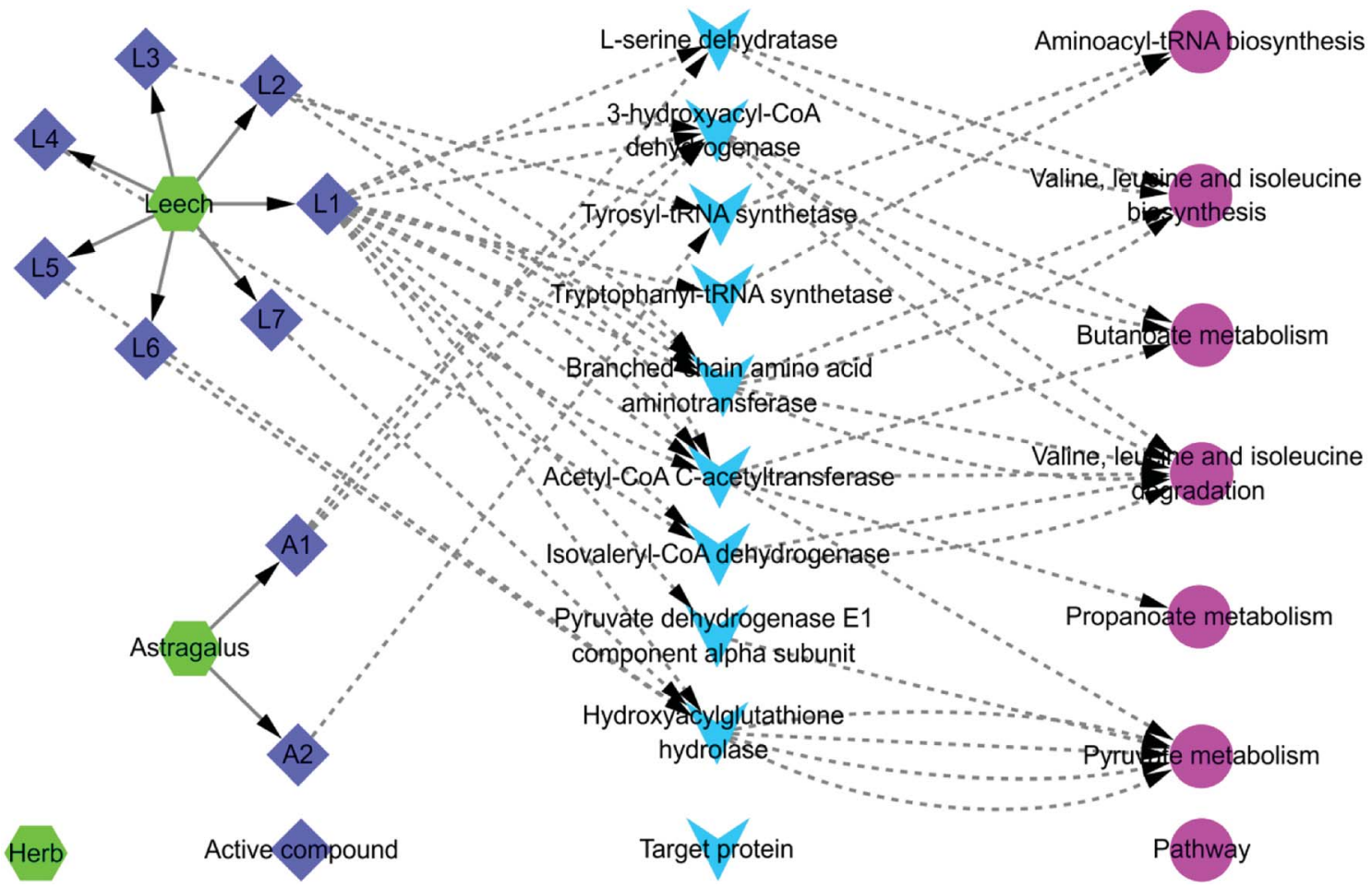

Fig. 7 The total interaction network diagram. A1: 3,9-di-O-methylnissolin; A2: $(6 a R, 11 a R)-9,10$-dimethoxy-6a,11a-dihydro-6H-benzofurano [3,2-c] chromen-3-ol; L1: hirudin; L2: L-isoleucine; L3: phenylalanine; L4: valine; L5: hirudinoidine A; L6: hirudinoidine B; L7: hirudinoidine C. Detailed information is available in ESI, Table S4.†

acetyl-CoA C-acetyltransferase [EC: 2.3.1.9]) to affect valine, leucine and isoleucine biosynthesis/degradation. Studies also have shown that administration of leucine or isoleucine can improve insulin sensitivity in mice with high-fat diets indicating that BCAAs can ameliorate insulin resistance through interactions in organs targeted by insulin..$^{75-77}$ Hence, we believe that Astragalus and Leech may alleviate T2DN by reducing gluconeogenesis of amino acids in the liver, and improving insulin sensitivity. The potential mechanism of action probably include increasing the liver $\mathrm{X}$ receptor/sterol regulatory element binding protein-1c pathway, suppressing hepatic expression of glucose-6-phosphatase, and the accelerated utility of glucose and glucose-6-phosphate in the liver. ${ }^{74,78}$ Besides, $(6 a R, 11 a R)$ 9,10-dimethoxy-6a,11a-dihydro-6H-benzofurano[3,2-c]

chromen-3-ol in Astragalus, and hirudin and phenylalanine in Leech can also have an effect on aminoacyl-tRNA biosynthesis via acting on tyrosyl-tRNA synthetase (TyrRS) [EC: 6.1.1.1] and tryptophanyl-tRNA synthetase (TrpRS) [EC: 6.1.1.2]. Study has shown that oxidative stress will induce TyrRS to rapidly translocate from the cytosol to the nucleus to protect against DNA damage. ${ }^{54,79}$ Whereas TrpRS have been proved be conformational isomers of homology to TyrRS. ${ }^{80}$ Meanwhile, Chou et al. ${ }^{81}$ have shown tryptophan catalyzed and activated by TrpRS might be regarded as a potential prognostic marker for diabetic nephropathy. Thus, this suggests that T2DN rats may have undergone oxidative stress, while Astragalus and Leech may reduce DNA damage caused by this oxidative stress through antioxidation.
What's more, taurine can be synthesized from methionine in the liver and removed mainly through renal excretion. Studies have shown that taurine possess the ability to maintain the structural integrity of cell membranes, and antioxidant activity. ${ }^{82}$ The level of taurine is decreased in T2DN group, but increase significantly in treated group, also manifesting that Astragalus and Leech are likely to have a certain therapeutic effects on T2DN rats via antioxidant activity. Glutamate is involved in the metabolism of toxic ammonia in vivo, and urea synthesis is influenced, the concentration of blood ammonia increases, thereby resulting in hyperammonemia when the liver and kidney function is impaired. ${ }^{54}$ Therefore, Astragalus and Leech should have a protective effect on the liver and kidney.

\section{Energy metabolism}

Succinate, a kind of TCA cycle intermediate, shows a decreased level in T2DN group, indicating TCA cycle is disordered and impairment of mitochondrial function. Besides, the declined level of lactate found in feces is indicative of decreased glycolysis. This indicates that TCA cycle and glycolysis may be inhibited, leading to energy compensation through the metabolism of energy storage substances and the enhancement of gluconeogenesis. Creatinine, a breakdown product of phosphocreatine in muscle, is transferred to the kidneys via the blood circulation. ${ }^{83}$ The levels of creatinine and glycerol decrease significantly in feces also indicate that the kidney may have been damaged. Thus, nonglycemic substances (e.g., lactate, glycerol, alanine, glutamate and valine) will supply energy to the body through the 
gluconeogenesis pathway. ${ }^{84}$ Study has been proved that gluconeogenesis is closely related to the pathogenesis of $\mathrm{DN}$, and ameliorate glucose metabolism by regulating gluconeogenesis is one of the important strategies against diabetic complications. ${ }^{69}$ All of these show that there is a significant disturbance of energy metabolism in T2DN rats. In contrast, the levels of lactate, succinate, creatinine, $\alpha$-glucose and glycerol are elevated significantly in treated group. Results of systems pharmacology analysis also show that hirudin and hirudinoidine A-C in Leech have an effect on pyruvate metabolism by acting on pyruvate dehydrogenase E1 component alpha subunit [EC: 1.2.4.1], hydroxyacylglutathione hydrolase [EC: 3.1.2.6] and acetyl-CoA Cacetyltransferase [EC: 2.3.1.9]. Pyruvate metabolism plays a pivotal role in the energy supply and the metabolic linkages of the three major nutrients (glucoses, fats and amino acids). As a result, Leech, to a certain extent, may play a therapeutic role by improving energy metabolism of T2DN rats.

\section{Ketone body and fatty acid metabolism}

3-Hydroxybutyrate is a product of ketone body and fatty acid metabolism in the liver's mitochondria. The analysis of fecal samples shows that the level of 3-hydroxybutyrate decrease significantly, suggesting a reduced activity of ketogenesis pathway or the presence of mitochondrial dysfunction in T2DN rats. Acetate is an important product of fatty acid $\beta$-oxidation and its decline significantly in feces of T2DN rats indicates reduced fatty acid $\beta$-oxidation. ${ }^{69}$ Acetate, $n$-butyrate and propionate, end products of the intestinal microbial fermentation of indigestible foods, are all short chain fatty acids (SCFAs) with potential anticarcinogenic activity of microbial metabolites present in feces. ${ }^{64}$ Notably, the levels of $n$-butyrate and propionate in T2DN rats increase significantly, but decrease after the treatment of Astragalus and Leech. It may be due to abnormal glucose metabolism and fatty acid metabolism in T2DN rats, which need to increase intestinal energy harvesting by means of SCFAs to supplement the energy. Meanwhile, 3,9-di-O-methylnissolin in Astragalus, and hirudin in Leech can affect butanoate metabolism and propanoate metabolism by acting on 3hydroxyacyl-CoA dehydrogenase and acetyl-CoA Cacetyltransferase, thereby reversing SCFAs to normal levels. A growing body of evidence suggests that SCFAs can beneficially modulate adipose tissue, skeletal muscle and liver tissue function, thereby contributing to the improvement of glucose homeostasis and insulin sensitivity. ${ }^{65}$ Furthermore, several mechanisms that link SCFAs to insulin sensitivity and the development of T2DM have been proposed, such as the interorgan effects on adipose tissue function and lipid storage capacity, inflammatory profile, and liver metabolism. ${ }^{\mathbf{8 5 - 8 8}}$ Thus, SCFAs, as potential metabolic targets to prevent and counteract obesity and its associated disorders in glucose metabolism and insulin resistance, have been paid more and more attention.

\section{Methylamine metabolism}

Trimethylamine (TMA) and dimethylamine (DMA), two kinds of methylamine metabolites, are important non-perturbing renal osmolytes produced via the degradation of choline to TMA and its di- and monoamine metabolites by the action of gut microflora. ${ }^{69,89}$ The decreased fecal concentrations of TMA and DMA in T2DN rats manifest the downregulation of intestinal flora activity and methylamine metabolism pathway. After the treatment of Astragalus and Leech, however, both of them are significantly increased, suggesting that Astragalus and Leech may be able to promote intestinal energy harvesting by increasing the activity of intestinal flora. Furthermore, asymmetric dimethylarginine (ADMA), a major precursor of DMA, can be hydrolyzed to DMA and L-citrulline by dimethylaminohydrolase (DDAH). What's more, the inhibition of DDAH has been proved to be a promising therapeutic strategy for the treatment of DN patients. ${ }^{69}$ The method for manipulating endogenous DMA concentrations, and regulating the endogenous DMA concentrations and the production of nitric oxide (NO) in conditions has been shown to contribute to illnesses. ${ }^{90}$ Hence, we hypothesize that Astragalus and Leech may also act on DDAH.

\section{Secondary bile acid metabolism and urea cycle}

Deoxycholate is one of the secondary bile acids, which is a metabolic byproduct of intestinal bacteria. Deoxycholate and other secondary bile acids will cause DNA damage. ${ }^{91}$ Secondary bile acids increase intracellular production of reactive oxygen and reactive nitrogen species resulting in increased oxidative stress and DNA damage. ${ }^{92,93}$ Additionally, diabetic individuals have been shown to suffer from dysregulation of bile acid (BA) homeostasis and dysbiosis of the intestinal microbiome. ${ }^{72}$ The level of deoxycholate in T2DN rats increase significantly, but decrease after the treatment of Astragalus and Leech. This shows that after injection of STZ, oxidative stress occurs in rats, while Astragalus and Leech may delay the development of diabetic nephropathy by reducing reactive oxygen species and DNA damage in T2DN rats.

Citrulline, precursor of arginine, is involved in urea cycle in liver's mitochondria. The level of citrulline in T2DM rats increase significantly, possibly due to the damage of mitochondria and the disorder of urea cycle. L-citrulline supplementation established an anti-inflammatory profile and significantly protects from kidney damage during type 1 diabetes. Moreover, study has proved that $\mathrm{L}$-citrulline can prevent diabetes mellitus-induced glomerular hyperfltration, proteinuria and kidney dysfunction in rat through promoting nitric oxide production. ${ }^{94}$ Thus, it may be due to the injection of STZ led to the occurrence of inflammatory reactions, the body needs a lot of citrulline to play an anti-inflammatory effect on kidney. After the treatment of Astragalus and Leech, however, the function of mitochondria and inflammation effect of T2DN rats was improved, so the level of citrulline decreased significantly. From Table $\mathrm{S} 4, \dagger$ it is also apparent that most target proteins (i.e. [EC: 1.1.1.35], [EC: 2.6.1.42], [EC: 2.3.1.9], [EC: 1.3.8.4], [EC: 1.2.4.1], [EC: 3.1.2.6]), acted by active compounds (A1, L1, L2, L4 - L7) from Astragalus and Leech, are existed in mitochondria. Consequently, it can also be demonstrated that Astragalus and Leech can treat T2DN by improving mitochondrial function. 
Overall, while the combination of Astragalus and Leech has been shown to be potentially effective, the outcomes of our study are limited as these two TCMs were not tested individually. Thus, it is necessary to add a separate administration group of Astragalus and Leech to analyze and verify the results of this study from the perspective of pharmacology and targeted metabonomics. In addition, synergistic effects among compounds from different herbs can't be predicted by the systems pharmacology approach as it can only predict the target of a single compound. Therefore, new and active compounds, produced by one or more TCMs' synergistic effects, may not be predictable. This is also a current limitation of system pharmacology. In the later period, we still need to further study the potential mechanism of T2DN treated by Astragalus and Leech, and verify the results of systems pharmacology from the aspects of pharmacology and cell biology. What's more, using NMR approach as the phenotype of the treatment (herbs, etc.), to some degree, may obscure the resonances of endogenic metabolites in a given biofluid, mainly urine, serum/plasma and feces. This is due to the intrinsically limitations of the low sensitivity of the NMR approach itself, and it may be better to combine higher sensitivity methods (e.g. UPLC-MS/MS, GCMS) to detect endogenic metabolites.

\section{Conclusions}

In summary, by combining systems pharmacology and fecal metabonomics, we revealed preliminarily the systemic metabolic changes during the development of T2DN and the therapeutic mechanism of Astragalus and Leech on T2DN rats. Results of systems pharmacology analysis revealed that 9 active compounds and 9 target proteins of Astragalus and Leech were closely associated with the treatment of T2DN. Using fecal metabonomics analysis, 22 biomarkers were eventually found to be closely associated with the occurrence of T2DN. Combined with systems pharmacology and fecal metabonomics, these biomarkers were found to be mainly associated with 6 pathways, involving amino acid metabolism, energy metabolism, ketone body and fatty acid metabolism, methylamine metabolism, and secondary bile acid metabolism and urea cycle. The underlying mechanisms of action include protection of the liver and kidney, enhancement of insulin sensitivity and antioxidant activity, and improvement of mitochondrial function. To the best of our knowledge, this is the first time that systems pharmacology combined with fecal metabonomics is being used to study T2DN. 6 metabolites ( $n$-butyrate, deoxycholate, propionate, tryptophan, taurine and glycerol) associated with T2DN were newly discovered in fecal samples. These 6 metabolites were mainly derived from the intestinal flora, and related to amino acid metabolism, fatty acid metabolism, and secondary bile acid metabolism. We hope the results of this study can be inspirational and helpful for further exploration of T2DN treatment. Meanwhile, our results highlight that explore biomarkers of T2DN and therapeutic mechanisms of TCM formulas on T2DN by combining systems pharmacology and fecal metabonomics methods are promising strategies.

\section{Conflicts of interest}

The authors declare no competing financial interest.

\section{Acknowledgements}

We acknowledge the financial supports from the National Nature Science Foundation of China (No. 81403153), and Science and Technology Planning Project of Guangdong Province, China (No. 2014A020212603).

\section{References}

1 F. Conserva, L. Gesualdo and M. Papale, J. Diabetes Res., 2016, 2016, 7934504.

2 J. L. Gross, M. J. de Azevedo, S. P. Silveiro, L. H. Canani, M. L. Caramori and T. Zelmanovitz, Diabetes Care, 2005, 28, 164-176.

3 Y. S. Kanwar, L. Sun, P. Xie, F. Liu and S. Chen, Annu. Rev. Pathol.: Mech. Dis., 2011, 6, 395-423.

4 D. K. Singh, P. Winocour and K. Farrington, Nat. Rev. Endocrinol., 2011, 7, 176-184.

5 S. Dronavalli, I. Duka and G. L. Bakris, Nat. Clin. Pract. Endocrinol. Metab., 2008, 4, 444-452.

6 S. Clotet, M. Riera, J. Pascual and M. J. Soler, Am. J. Physiol. Renal Physiol., 2016, 310, F945-F957.

7 G. H. Tesch and T. J. Allen, Nephrology, 2007, 12, 261-266.

8 S. Rezzi, Z. Ramadan, L. B. Fay and S. Kochhar, J. Proteome Res., 2007, 6, 513-525.

9 J. K. Nicholson and J. C. Lindon, Nature, 2008, 455, 10541056.

10 T. J. Wang, M. G. Larson, R. S. Vasan, S. Cheng, E. P. Rhee, E. McCabe, G. D. Lewis, C. S. Fox, P. F. Jacques, C. Fernandez, C. J. O'Donnell, S. A. Carr, V. K. Mootha, J. C. Florez, A. Souza, O. Melander, C. B. Clish and R. E. Gerszten, Nat. Med., 2011, 17, 448-453.

11 J. Liu, C. Wang, F. Liu, Y. Lu and J. Cheng, Anal. Bioanal. Chem., 2015, 407, 2569-2579.

12 J. Zhang, L. Yan, W. Chen, L. Lin, X. Song, X. Yan, W. Hang and B. Huang, Anal. Chim. Acta, 2009, 650, 16-22.

13 M. Li, X. Wang, J. Aa, W. Qin, W. Zha, Y. Ge, L. Liu, T. Zheng, B. Cao, J. Shi, C. Zhao, X. Wang, X. Yu, G. Wang and Z. Liu, Am. J. Physiol. Renal Physiol., 2013, 304, F1317-F1324.

14 C. Barrios, T. D. Spector and C. Menni, Arch. Biochem. Biophys., 2016, 589, 81-92.

15 J. R. Marchesi, E. Holmes, F. Khan, S. Kochhar, P. Scanlan, F. Shanahan, I. D. Wilson and Y. Wang, J. Proteome Res., 2007, 6, 546-551.

16 M. De Angelis, E. Montemurno, M. Piccolo, L. Vannini, G. Lauriero, V. Maranzano, G. Gozzi, D. Serrazanetti, G. Dalfino, M. Gobbetti and L. Gesualdo, PLoS One, 2014, 9, e99006.

17 T. Zhao, H. Zhang, X. Zhang, T. Zhao, H. Y. Lan, Q. Liang, G. Luo and P. Li, J. Ethnopharmacol., 2015, 166, 31-41.

18 H. B. Xu, R. H. Jiang, X. Z. Chen and L. Li, J. Ethnopharmacol., 2012, 143, 701-708. 
19 M. Li, W. Wang, J. Xue, Y. Gu and S. Lin, J. Ethnopharmacol., 2011, 133, 412-419.

20 R. Jin, B. Zhang, C. M. Xue, S. M. Liu, Q. Zhao and K. Li, Zhong Xi Yi Jie He Xue Bao., 2011, 9, 665-674.

21 F. Wu and X. Chen, Zhongyaocai, 2004, 27, 232-234.

22 M. Xiao and J. M. Fan, Medical Journal, 2009, 21, 474-475.

23 W. Deng and J. A. Fang, Chinese Journal of Integrated Traditional and Western Nephrology, 2012, 13, 571-574.

24 E. Song, M. Liu and R. Jia, Research of integrated traditional Chinese and Western medicine, 2009, 1, 126-129.

25 J. Zhang, X. Xie, C. Li and P. Fu, J. Ethnopharmacol., 2009, 126, 189-196.

26 H. Liao, L. Hu, X. Cheng, X. Wang, J. Li, L. Banbury and R. Li, J. Immunol. Res., 2017, 2017, 3780572.

27 Y. M. Liu, J. Zhang, F. Shi and A. Liu, China Journal of Information on Traditional Chinese Medicine., 2011, 18, 108110.

28 H. Dong, J. X. Ren, J. J. Wang, L. S. Ding, J. J. Zhao, S. Y. Liu and H. M. Gao, Evid.-Based Complementary Altern. Med., 2016, 2016, 7895935.

29 X. Liu, M. F. Gao and Y. Kong, Pharm. Biotechnol., 2017, 24, 76-80.

30 J. P. Hildebrandt and S. Lemke, Naturwissenschaften, 2011, 98, 995-1008.

31 W. Y. Liang, Jilin Journal of Chinese Medicine, 2005, 25, 58-59.

32 B. F. Hu, L. S. Meng, M. Huang, Q. Y. Li and X. Q. Ren, Liaoning J. Tradit. Chin. Med., 2003, 30, 238.

33 M. Z. Jiang, T. P. Tang and X. Z. Ren, Liaoning J. Tradit. Chin. Med., 2017, 44, 193-196.

34 G. Y. Wang, MPhil thesis, Nanjing University of Chinese Medicine, 2015.

35 X. J. Fu and R. B. Xiong, Chin. Arch. Tradit. Chin. Med., 2013, 31, 305-308.

36 X. J. Fu, H. C. Zhang, Y. F. Hu, H. G. Qian and J. Liu, Clin. Med., 2004, 24, 47-48.

37 X. J. Fu and R. B. Xiong, Journal of Emergency in Traditional Chinese Medicine, 2012, 21, 1595-1597.

38 S. S. Chen and X. Z. Ren, J. Liaoning Univ. Tradit. Chin. Med., 2012, 14, 447-462.

39 C. Huang, C. Zheng, Y. Li, Y. Wang, A. Lu and L. Yang, Briefings Bioinf., 2014, 15, 710-733.

40 A. Zhang, H. Fang, Y. Wang, G. Yan, H. Sun, X. Zhou, Y. Wang, L. Liu and X. Wang, $R S C A d v ., 2017,7,51069-$ 51078.

41 W. N. Zhang, A. P. Li, Y. S. Qi, X. M. Qin and Z. Y. Li, J. Pharm. Biomed. Anal., 2018, 158, 128-136.

42 H. Q. Pang, S. J. Yue, Y. P. Tang, Y. Y. Chen, Y. J. Tan, Y. J. Cao, X. Q. Shi, G. S. Zhou, A. Kang, S. L. Huang, Y. J. Shi, J. Sun, Z. S. Tang and J. A. Duan, Front. Pharmacol., 2018, 9, 165.

43 J. Ru, P. Li, J. Wang, W. Zhou, B. Li, C. Huang, P. Li, Z. Guo, W. Tao and Y. Yang, J. Cheminf., 2014, 6, 13.

44 X. Xu, W. Zhang, C. Huang, Y. Li, H. Yu, Y. Wang, J. Duan and Y. Ling, Int. J. Mol. Sci., 2012, 13, 6964-6982.

45 W. Tao, X. Xu, X. Wang, B. Li, Y. Wang, Y. Li and L. Yang, J. Ethnopharmacol., 2013, 145, 1-10.

46 D. E. Clark, Drug Discovery Today, 2003, 8, 927-933.
47 Y. B. Li, Y. Xiang, W. H. Huang and N. Yao, Strait Pharmaceutical Journal (Haixia Yaoxue), 2009, 21, 75-77.

48 X. Guo, J. Sun and H. Zhang, Jilin J. Tradit. Chin. Med., 2015, 35, 47-49.

49 W. G. Jing, F. U. Jiang, Y. M. Liu and A. Liu, Chin. J. Exp. Tradit. Med. Formulae, 2014, 20, 120-123.

50 D. Han, J. X. Ren, J. J. Wang, L. S. Ding, J. J. Zhao, S. Y. Liu and H. M. Gao, Evid.-Based Complementary Altern. Med., 2016, 2016, 1-11.

51 Y. B. Li, W. H. Huang and Y. Xiang, Helv. Chim. Acta, 2010, 91, 303-307.

52 X. Wang, Y. Shen, S. Wang, S. Li, W. Zhang, X. Liu, L. Lai, J. Pei and H. Li, Nucleic Acids Res., 2017, 45, W356-W360.

53 A. M. Zidan, A. A. Hemeida, A. M. Hussein and M. H. Hashim, Int. Res. J. Pharm., 2013, 4, 37-39.

54 R. Chen, J. Wang, C. Liao, L. Zhang, Q. Guo and X. Wang, RSC Adv., 2018, 8, 1098-1115.

55 X. J. Fu and R. B. Xiong, Journal of Emergency in Traditional Chinese Medicine, 2012, 21, 1595-1597.

56 I. O. Sherif, J. Physiol. Biochem., 2014, 70, 961-969.

57 M. A. Morsy, G. H. Heeba and M. E. Mahmoud, Eur. J. Pharmacol., 2015, 750, 90-97.

58 M. J. Reed, K. Meszaros, L. J. Entes, M. D. Claypool, J. G. Pinkett, T. M. Gadbois and G. M. Reaven, Metabolism, 2000, 49, 1390-1394.

59 F. Tian, L. Gu, A. Si, Q. Yao, X. Zhang, J. Zhao and D. Hu, J. Chromatogr. B: Anal. Technol. Biomed. Life Sci., 2015, 1007, 140-148.

60 Y. Zhao, J. Wu, J. V. Li, N.-Y. Zhou, H. Tang and Y. Wang, J. Proteome Res., 2013, 12, 2987-2999.

61 J. Xia and D. S. Wishart, Nat. Protoc., 2011, 6, 743-760.

62 L. Zhang, Y. Ye, Y. An, Y. Tian, Y. Wang and H. Tang, J. Proteome Res., 2011, 10, 614-623.

63 G. Le Gall, S. O. Noor, K. Ridgway, L. Scovell, C. Jamieson, I. T. Johnson, I. J. Colquhoun, E. K. Kemsley and A. Narbad, J. Proteome Res., 2011, 10, 4208-4218.

64 J. Saric, Y. Wang, J. Li, M. Coen, J. Utzinger, J. R. Marchesi, J. Keiser, K. Veselkov, J. C. Lindon, J. K. Nicholson and E. Holmes, J. Proteome Res., 2008, 7, 352-360.

65 D. M. Jacobs, N. Deltimple, E. van Velzen, F. A. van Dorsten, M. Bingham, E. E. Vaughan and J. van Duynhoven, NMR Biomed., 2008, 21, 615-626.

66 S. Zhang, G. A. Nagana Gowda, V. Asiago, N. Shanaiah, C. Barbas and D. Raftery, Anal. Biochem., 2008, 383, 76-84.

67 L. Zhao, H. Gao, F. Lian, X. Liu, Y. Zhao and D. Lin, Am. J. Physiol. Renal Physiol., 2011, 300, F947-F956.

68 A. Hirayama, E. Nakashima, M. Sugimoto, S. Akiyama, W. Sato, S. Maruyama, S. Matsuo, M. Tomita, Y. Yuzawa and T. Soga, Anal. Bioanal. Chem., 2012, 404, 3101-3109.

69 T. Wei, L. Zhao, J. Jia, H. Xia, Y. Du, Q. Lin, X. Lin, X. Ye, Z. Yan and H. Gao, Sci. Rep., 2015, 5, 11998.

70 J. Liu, D. Wang, Y. Chen, H. Sun, S. He, C. Wang, G. Yang, M. Shi, J. Zhang, Y. Ren, L. Wang, Y. Lu and J. Cheng, Mol. BioSyst., 2013, 9, 2645-2652.

71 M. Ala-Korpela, Expert Rev. Mol. Diagn., 2007, 7, 761-773. 
72 S. Pal, S. J. Polyak, N. Bano, W. C. Qiu, R. L. Carithers, M. Shuhart, D. R. Gretch and A. Das, J. Gastroenterol. Hepatol., 2010, 25, 627-634.

73 T. Tsukishiro, Y. Shimizu, K. Higuchi and A. Watanabe, J. Gastroenterol. Hepatol., 2000, 15, 849-859.

74 K. Tajiri and Y. Shimizu, World J. Gastroenterol., 2013, 19, 7620-7629.

75 Y. Zhang, K. Guo, R. E. LeBlanc, D. Loh, G. J. Schwartz and Y. H. Yu, Diabetes, 2007, 56, 1647-1654.

76 O. Ikehara, N. Kawasaki, K. Maezono, M. Komatsu and A. Konishi, Biol. Pharm. Bull., 2008, 31, 469-472.

77 T. Kawaguchi, Y. Nagao, H. Matsuoka, T. Ide and M. Sata, Int. J. Mol. Med., 2008, 22, 105-112.

78 N. Higuchi, M. Kato, M. Miyazaki, M. Tanaka, M. Kohjima, T. Ito, M. Nakamuta, M. Enjoji, K. Kotoh and R. Takayanagi, J. Cell. Biochem., 2011, 112, 30-38.

79 N. Wei, Y. Shi, L. N. Truong, K. M. Fisch, T. Xu, E. Gardiner, G. Fu, Y. S. Hsu, S. Kishi, A. I. Su, X. Wu and X. L. Yang, Mol. Cell, 2014, 56, 323-332.

80 S. Doublie, G. Bricogne, C. Gilmore and C. W. Carter Jr, Structure, 1995, 3, 17-31.

81 C. A. Chou, C. N. Lin, D. T. Chiu, I. W. Chen and S. T. Chen, J. Diabetes Invest., 2018, 9, 366-374.

82 J. Y. Wu and H. Prentice, J. Biomed. Sci., 2010, 17, 1-6.

83 J. S. Tian, G. J. Peng, X. X. Gao, Y. Z. Zhou, J. Xing, X. M. Qin and G. H. Du, J. Ethnopharmacol., 2014, 158(Pt A), 1-10.
84 R. Chen, J. Wang, C. Liao, N. Ma, L. Zhang and X. Wang, RSC Adv., 2017, 7, 34251-34261.

85 E. E. Canfora, J. W. Jocken and E. E. Blaak, Nat. Rev. Endocrinol., 2015, 11, 577-591.

86 G. den Besten, A. Bleeker, A. Gerding, K. van Eunen, R. Havinga, T. H. van Dijk, M. H. Oosterveer, J. W. Jonker, A. K. Groen, D. J. Reijngoud and B. M. Bakker, Diabetes, 2015, 64, 2398-2408.

87 S. Al-Lahham, H. Roelofsen, F. Rezaee, D. Weening, A. Hoek, R. Vonk and K. Venema, Eur. J. Clin. Invest., 2012, 42, 357364.

88 E. F. Murphy, P. D. Cotter, S. Healy, T. M. Marques, O. O'Sullivan, F. Fouhy, S. F. Clarke, P. W. O'Toole, E. M. Quigley, C. Stanton, P. R. Ross, R. M. O'Doherty and F. Shanahan, Gut, 2010, 59, 1635-1642.

89 J. Messenger, S. Clark, S. Massick and M. Bechtel, J. Clin. Aesthet. Dermatol., 2013, 6, 45-48.

90 S. J. Brinkmann, M. C. de Boer, N. Buijs and P. A. van Leeuwen, Curr. Opin. Clin. Nutr. Metab. Care, 2014, 17, 90-97.

91 H. Bernstein, C. Bernstein, C. M. Payne, K. Dvorakova and H. Garewal, Mutat. Res., 2005, 589, 47-65.

92 J. Tsuei, T. Chau, D. Mills and Y. J. Wan, Exp. Biol. Med., 2014, 239, 1489-1504.

93 H. Ajouz, D. Mukherji and A. Shamseddine, World J. Surg. Oncol., 2014, 12, 164.

94 P. Persson, A. Fasching, T. Teerlink, P. Hansell and F. Palm, Hypertension, 2014, 64, 323-329. 